\title{
HOMOGENIZATION FOR NON-SELF-ADJOINT PERIODIC ELLIPTIC OPERATORS ON AN INFINITE CYLINDER
}

\author{
NIKITA N. SENIK
}

\begin{abstract}
We consider the problem of homogenization for non-self-adjoint second-order elliptic differential operators $\mathcal{A}^{\varepsilon}$ of divergence form on $L_{2}\left(\mathbb{R}^{d_{1}} \times\right.$ $\mathbb{T}^{d_{2}}$ ), where $d_{1}$ is positive and $d_{2}$ is non-negative. The coefficients of the operator $\mathcal{A}^{\varepsilon}$ are periodic in the first variable with period $\varepsilon$ and smooth in a certain sense in the second. We show that, as $\varepsilon$ gets small, $\left(\mathcal{A}^{\varepsilon}-\mu\right)^{-1}$ and $D_{x_{2}}\left(\mathcal{A}^{\varepsilon}-\mu\right)^{-1}$ converge in the operator norm to, respectively, $\left(\mathcal{A}^{0}-\mu\right)^{-1}$ and $D_{x_{2}}\left(\mathcal{A}^{0}-\mu\right)^{-1}$, where $\mathcal{A}^{0}$ is an operator whose coefficients depend only on $x_{2}$. We also obtain an approximation for $D_{x_{1}}\left(\mathcal{A}^{\varepsilon}-\mu\right)^{-1}$ and find the next term in the approximation for $\left(\mathcal{A}^{\varepsilon}-\mu\right)^{-1}$. Estimates for the rates of convergence and the rates of approximation are provided and are sharp with respect to the order.
\end{abstract}

\section{INTRODUCTION}

The periodic homogenization problem consists in studying asymptotic behavior of solutions of differential equations with rapidly oscillating coefficients. The key fact about homogenization is that the solutions of such problems converge to solutions of problems whose coefficients no longer oscillate; in applications, this means that we approximate highly heterogeneous media by a homogenous one. Classical arguments (as in $[\mathrm{BLP}],[\mathrm{BP}]$ or $[\mathrm{ZHKO}]$ ) show that the convergence is weak or strong. In certain cases, they may even imply the norm-resolvent convergence of the corresponding operators (see [OSHY]); however, the question of whether these operators converge in the norm-resolvent sense without any additional regularity assumptions remained open until 2001, when BIRMAN and SusLina BSU1, (see also BSU2]) proved that this is the case for a broad class of elliptic problems. Many related results appeared in subsequent years; see, for example, GR1], GR2], [ZH], $\mathrm{ZHP},[\mathrm{BO}, \overline{\mathrm{KLS}}$ and references therein. In the recent paper $\mathrm{CHC}$, a result of this kind was obtained for some of the high-contrast problems.

The present paper is motivated by the study of homogenization problems for operators whose coefficients are periodic only in certain directions. These arise naturally in many applications - for instance, in the theory of waveguides and in elasticity, - and were investigated in $[\mathrm{S}-\mathrm{HT}], \mathrm{OSHY}],[\mathrm{Su} 1,[\mathrm{BCSU}]$ and $[\mathrm{SE1}$.

2010 Mathematics Subject Classification. 35B27.

Key words and phrases. homogenization, operator error estimates, periodic differential operators, effective operator, corrector.

The author was supported by SPbSU grant 0.38.237.2014 and by RFBR grant 14-01-00760.

St. Petersburg State University, Universitetskaya nab. 7/9, St. Petersburg 199034, Russia (N.N.Senik@gmail.com). 
Let $\Xi$ be the cylinder $\mathbb{R}^{d_{1}} \times \mathbb{T}^{d_{2}}$. In [SU1], SusLINA studied the homogenization problem for elliptic self-adjoint operators $\mathcal{A}^{\varepsilon}$ on $\Xi$ of the form

$$
\mathcal{A}^{\varepsilon}=D_{1}^{*} A_{11}\left(\varepsilon^{-1} x_{1}, x_{2}\right) D_{1}+D_{2}^{*} A_{22}\left(\varepsilon^{-1} x_{1}, x_{2}\right) D_{2} .
$$

Here, $A_{11}$ and $A_{22}$ are periodic in the first variable and Lipschitz in the second. She proved that $\mathcal{A}^{\varepsilon}$ converges in the norm-resolvent sense to an operator $\mathcal{A}^{0}$, whose coefficients depend only on the non-periodic variable $x_{2}$, and furthermore

$$
\left\|\left(\mathcal{A}^{\varepsilon}+1\right)^{-1}-\left(\mathcal{A}^{0}+1\right)^{-1}\right\|_{\mathbf{B}\left(L_{2}(\Xi)\right)} \leq C \varepsilon
$$

Such problems were further analyzed in SE1], where we extended that result to self-adjoint operators with lower-order terms and also obtained an approximation for the resolvent in $\mathbf{B}\left(L_{2}(\Xi), H^{1}(\Xi)\right.$ ). (Strictly speaking, the work [SE1] deals with the case $d_{1}=d_{2}=1$, although it is possible to use the techniques of that article to treat the other cases.) But operators with non-diagonal terms were left beyond the scope of these papers, and it is our intention here to fill this gap.

In this article, we will be concerned with an elliptic non-self-adjoint operator $\mathcal{A}^{\varepsilon}$ on $\Xi$ of the form

$$
\mathcal{A}^{\varepsilon}=D^{*} A\left(\varepsilon^{-1} x_{1}, x_{2}\right) D+D^{*} a_{1}\left(\varepsilon^{-1} x_{1}, x_{2}\right)+a_{2}^{*}\left(\varepsilon^{-1} x_{1}, x_{2}\right) D+q\left(\varepsilon^{-1} x_{1}, x_{2}\right),
$$

where $A, a_{1}, a_{2}$ and $q$ are periodic in the first variable with respect to a lattice in $\mathbb{R}^{d_{1}}$ and have weak derivatives with respect to the second variable. We further assume that the coefficients together with the derivatives belong to certain spaces of Sobolev multipliers. We find approximations for $\left(\mathcal{A}^{\varepsilon}-\mu\right)^{-1}$ and $D\left(\mathcal{A}^{\varepsilon}-\mu\right)^{-1}$ in the operator norm and prove the following estimates:

$$
\begin{gathered}
\left\|\left(\mathcal{A}^{\varepsilon}-\mu\right)^{-1}-\left(\mathcal{A}^{0}-\mu\right)^{-1}\right\|_{\mathbf{B}\left(L_{2}(\Xi)\right)} \leq C \varepsilon, \\
\left\|D_{2}\left(\mathcal{A}^{\varepsilon}-\mu\right)^{-1}-D_{2}\left(\mathcal{A}^{0}-\mu\right)^{-1}\right\|_{\mathbf{B}\left(L_{2}(\Xi)\right)^{d_{2}}} \leq C \varepsilon
\end{gathered}
$$

and

$$
\begin{gathered}
\left\|D_{1}\left(\mathcal{A}^{\varepsilon}-\mu\right)^{-1}-D_{1}\left(\mathcal{A}^{0}-\mu\right)^{-1}-\varepsilon D_{1} \mathcal{K}_{\mu}^{\varepsilon}\right\|_{\mathbf{B}\left(L_{2}(\Xi)\right)^{d_{1}}} \leq C \varepsilon, \\
\left\|\left(\mathcal{A}^{\varepsilon}-\mu\right)^{-1}-\left(\mathcal{A}^{0}-\mu\right)^{-1}-\varepsilon \mathcal{C}_{\mu}^{\varepsilon}\right\|_{\mathbf{B}\left(L_{2}(\Xi)\right)} \leq C \varepsilon^{2}
\end{gathered}
$$

(see the statements of Theorems $1-3$ in Section 3). Here, $\mathcal{A}^{0}$ is the effective operator and $\mathcal{K}_{\mu}^{\varepsilon}$ and $\mathcal{C}_{\mu}^{\varepsilon}$ are correctors. The effective operator has a form similar to that of $\mathcal{A}^{\varepsilon}$, with coefficients depending only on the non-periodic variable $x_{2}$, while the correctors involve rapidly oscillating functions. The estimates are sharp with respect to the order and the constants on the right may be expressed explicitly in terms of the problem parameters. Some of our results were announced in [SE2].

The estimates (2) and (4) have no analogue in SU1 and [SE1]. The former is new and the latter has appeared for the first time in BSU3, for certain self-adjoint operators on the entire space. A more recent development [SU4 has extended that result to self-adjoint operators with lower-order terms. We also mention here the paper $\left[\mathrm{P}\right.$, where an estimate similar to (4) was obtained for operators on $\mathbb{R}^{d}$ with smooth coefficients. However, all those results apply only to purely periodic operators.

On the other hand, we may regard the problem we address here as a special case of more general locally periodic homogenization problems (where the coefficients may depend on both $x$ and $\varepsilon^{-1} x$ ). From this point of view, estimates of type (1) and (3) are known; see, for instance, $[\mathrm{PT}]$, where symmetric operators with no lower-order terms were treated. In contrast, the estimate $(2)$ is a feature of our 
problem. As for (4), we believe that the arguments provided here can be used to prove a similar result for locally periodic operators as well.

The operator-theoretic method of BIRMAN and SusLina deals only with purely periodic operators and cannot be extended to locally-periodic ones. Nevertheless, the abstract results they obtained may be adapted, by ad hoc means, to get the approximations for operators with $A$ having block-diagonal structure, as shown in SU1 and SE1. However, operators with more general $A$ do not fit into this framework. So, if we are to handle these cases, we must develop a different approach.

Our program is as follows. We first reduce the problem to a problem on a fundamental domain for the lattice. This is done by applying the scaling transformation and the Gelfand transform, both with respect to the periodic variable. This step is identical to the one in [SU1] and [SE1]. The next step differs significantly. We prove suitable versions of the resolvent identity (see (63) and (67)). This enables us to verify the desired inequalities by elementary means.

Note that the torus $\mathbb{T}^{d_{2}}$ can be replaced by any flat manifold without boundary $\left(\mathbb{R}^{d_{2}}\right.$, for instance). We hope that the techniques presented in this article will prove useful in studying homogenization problems on domains of type $\mathbb{R}^{d_{1}} \times(0,1)^{d_{2}}$ with Dirichlet or Neumann boundary conditions as well.

The paper is organized as follows. In Section 2, we give the necessary background information, introduce the operator $\mathcal{A}^{\varepsilon}$, as well as the effective operator and the correctors, formulate the problem under consideration and provide an example of $\mathcal{A}^{\varepsilon}$. Section 3 contains presentation of the main results. In Section 4 we deal with the problem on the fundamental domain and prove the results.

\section{BASIC DEFINITIONS AND PROBLEM FORMULATION}

We begin with some notation.

2.1. Preliminaries. The symbol $\|\cdot\|_{U}$ denotes the norm on a normed space $U$. Let $U$ and $V$ be Banach spaces. We use the notation $\mathbf{B}(U, V)$ to denote the Banach space of bounded linear operators from $U$ to $V$. When $U=V$, the space $\mathbf{B}(U)=$ $\mathbf{B}(U, U)$ becomes a Banach algebra with identity $\mathcal{I}$. The inner product on a preHilbert space $U$ is denoted by $(\cdot, \cdot)_{U}$. In the finite-dimensional case $U=\mathbb{C}^{n}$, the norm and the inner product are denoted by $|\cdot|$ and $\langle\cdot, \cdot\rangle$, respectively. We shall identify the spaces $\mathbf{B}\left(\mathbb{C}^{n}, \mathbb{C}^{m}\right)$ with $\mathbb{C}^{m \times n}$.

Let $\Sigma$ be a domain in $\mathbb{R}^{d}$ and $U$ a Banach space. Then $L_{p}(\Sigma ; U)$, with $1 \leq p \leq \infty$, is the Banach space of strongly measurable functions $u: \Sigma \rightarrow U$ satisfying

$$
\|u\|_{L_{p}(\Sigma ; U)}=\left(\int_{\Sigma}\|u(x)\|_{U}^{p} d x\right)^{1 / p}<\infty
$$

if $p<\infty$ and

$$
\|u\|_{L_{\infty}(\Sigma ; U)}=\underset{x \in \Sigma}{\operatorname{ess} \sup }\|u(x)\|_{U}<\infty
$$

if $p=\infty$. In case $U=\mathbb{C}^{n}$, we shall write $\|\cdot\|_{p, \Sigma}$ for the norm on $L_{p}(\Sigma)^{n}=$ $L_{p}(\Sigma ; U)$ and $(\cdot, \cdot)_{2, \Sigma}$ for the inner product on $L_{2}(\Sigma)^{n}=L_{2}(\Sigma ; U)$. We denote by $W_{p}^{m}(\Sigma)$, with $m \in \mathbb{N}$ and $1 \leq p \leq \infty$, the Banach space of those measurable functions $u: \Sigma \rightarrow \mathbb{C}$ that possess all weak derivatives up to and including order $m$ and such that 


$$
\|u\|_{m, p, \Sigma}=\|u\|_{W_{p}^{m}(\Sigma)}=\left(\sum_{|\alpha| \leq m}\left\|D^{\alpha} u\right\|_{L_{p}(\Sigma)}^{p}\right)^{1 / p}<\infty
$$

if $p<\infty$ and

$$
\|u\|_{m, \infty, \Sigma}=\|u\|_{W_{\infty}^{m}(\Sigma)}=\max _{|\alpha| \leq m}\left\|D^{\alpha} u\right\|_{L_{\infty}(\Sigma)}<\infty
$$

if $p=\infty$. The Hilbert space $W_{2}^{m}(\Sigma)$ is denoted by $H^{m}(\Sigma)$, and $H^{m}(\Sigma)^{*}$ is its dual space under the pairing $(\cdot, \cdot)_{2, \Sigma}$. If $\Sigma$ is not open, then $W_{p}^{m}(\Sigma)$ will be understood to mean the Sobolev space on the interior of $\Sigma$.

Multipliers between Sobolev spaces are (generalized) functions such that the corresponding multiplication operators are bounded. Here we shall be brief; a thorough treatment of Sobolev multipliers may be found in $[\mathrm{MSH}$. Let $\Sigma$ be a Lipschitz domain in $\mathbb{R}^{d}$, and let $m$ and $n$ be non-negative integers satisfying $m \geq n$. Then $\gamma$ is a Sobolev multiplier between $H^{m}(\Sigma)$ and $H^{n}(\Sigma)$ (written $\gamma \in \mathbf{M}\left(H^{m}(\Sigma), H^{n}(\Sigma)\right)$ ) provided that the operator of multiplication $\gamma: H^{m}(\Sigma) \rightarrow H^{n}(\Sigma)$ is continuous. The space $\mathbf{M}\left(H^{m}(\Sigma), H^{n}(\Sigma)^{*}\right)$ of Sobolev multipliers between $H^{m}(\Sigma)$ and the dual of $H^{n}(\Sigma)$ is defined in the same way. Notice that an element of $\mathbf{M}\left(H^{m}(\Sigma), H^{n}(\Sigma)^{*}\right)$ is a complex-valued distribution.

We shall normally write $\|\gamma\|_{\mathbf{M}}$ for the norm of a Sobolev multiplier $\gamma$. This should lead to no confusion, since, once we discover that $\gamma \in \mathbf{M}\left(H^{m}(\Sigma), H^{n}(\Sigma)\right)$ (or $\gamma \in$ $\mathbf{M}\left(H^{m}(\Sigma), H^{n}(\Sigma)^{*}\right)$ ), we fix the spaces $H^{m}(\Sigma)$ and $H^{n}(\Sigma)$ (or the spaces $H^{m}(\Sigma)$ and $\left.H^{n}(\Sigma)^{*}\right)$.

Given a positive $\delta$, the scaling transformation $\mathcal{S}^{\delta}$ is defined to be the map that assigns to each measurable function $u$ on $\Sigma$ the measurable function $v$ on $\delta^{-1} \Sigma$ given by $v(y)=\delta^{d / 2} u(\delta y)$. Then $\mathcal{S}^{\delta}$ is an isomorphism of $H^{m}(\Sigma)$ onto $H^{m}\left(\delta^{-1} \Sigma\right)$, with

$$
\left\|\mathcal{S}^{\delta}\right\|_{\mathbf{B}\left(H^{m}(\Sigma), H^{m}\left(\delta^{-1} \Sigma\right)\right)} \leq \max \left\{1, \delta^{m}\right\},
$$

and an isometry provided that $m=0$. By duality $\mathcal{S}^{\delta}$ extends to $H^{m}(\Sigma)^{*}$, so that $\mathcal{S}^{\delta}: H^{m}(\Sigma)^{*} \rightarrow H^{m}\left(\delta^{-1} \Sigma\right)^{*}$ is also an isomorphism and

$$
\left\|\mathcal{S}^{\delta}\right\|_{\mathbf{B}\left(H^{m}(\Sigma)^{*}, H^{m}\left(\delta^{-1} \Sigma\right)^{*}\right)} \leq \max \left\{1, \delta^{-m}\right\} .
$$

Let $\left\{\lambda_{m}\right\}_{m \in[d]}$ be a basis of $\mathbb{R}^{d}$. Here, $[d]$ denotes the set of integers $\{1,2, \ldots, d\}$. Then the basis generates the lattice

$$
\Lambda=\left\{\lambda \in \mathbb{R}^{d}: \lambda=\sum_{m \in[d]} n_{m} \lambda_{m}, n_{m} \in \mathbb{Z}\right\}
$$

with the basic cell

$$
\Omega=\left\{x \in \mathbb{R}^{d}: x=\sum_{m \in[d]} x_{m} \lambda_{m}, x_{m} \in\left[-2^{-1}, 2^{-1}\right)\right\} .
$$

The dual lattice $\Lambda^{*}$ is generated by the basis $\left\{\lambda_{m}^{*}\right\}_{m \in[d]}$ that is defined by the equations $\left\langle\lambda_{m}, \lambda_{n}^{*}\right\rangle=2 \pi \delta_{m n}$. We denote the Brillouin zone by $\Omega^{*}$ :

$$
\Omega^{*}=\left\{k \in \mathbb{R}^{d}:|k|<\left|k-\lambda^{*}\right|, \lambda^{*} \in \Lambda^{*} \backslash\{0\}\right\} .
$$

Notice that the closure of $\Omega^{*}$ is a convex polyhedron containing the ball of radius $r_{\Lambda}=2^{-1} \min _{\lambda^{*} \in \Lambda^{*} \backslash\{0\}}\left|\lambda^{*}\right|$ centered at the origin. 
Lattices are intimately related to Fourier series. If $u$ is any function in $L_{2}(\Omega)$, then there is a unique representation

$$
u(x)=|\Omega|^{-1 / 2} \sum_{\lambda^{*} \in \Lambda^{*}} \hat{u}_{\lambda^{*}} e^{-i\left\langle x, \lambda^{*}\right\rangle},
$$

where the series converges in $L_{2}(\Omega)$. The corresponding mapping $u \mapsto\left\{\hat{u}_{\lambda^{*}}\right\}_{\lambda^{*} \in \Lambda^{*}}$ is an isometric isomorphism of $L_{2}(\Omega)$ onto $l_{2}\left(\Lambda^{*}\right)$.

Let $\widetilde{W}_{p}^{m}(\Omega)$ denote the subspace of $W_{p}^{m}(\Omega)$ consisting of functions whose periodic extensions are in $W_{p \text {,loc }}^{m}\left(\mathbb{R}^{d}\right)$. The symbol $\widetilde{W}_{p, 0}^{m}(\Omega)$ stands for the space of functions in $\widetilde{W}_{p}^{m}(\Omega)$ with zero mean value. We shall write $\widetilde{H}^{m}(\Omega)$ and $\widetilde{H}_{0}^{m}(\Omega)$ for $\widetilde{W}_{2}^{m}(\Omega)$ and $\widetilde{W}_{2,0}^{m}(\Omega)$. Observe that, for each $k \in \mathbb{R}^{d}$ and any $u \in \widetilde{H}^{1}(\Omega)$, we have

$$
\|(D+k) u\|_{2, \Omega}^{2}=\sum_{\lambda^{*} \in \Lambda^{*}}\left|\lambda^{*}+k\right|^{2}\left|\hat{u}_{\lambda^{*}}\right|^{2},
$$

which yields a variant of Poincaré's inequality:

$$
\left\|u-u_{\Omega}\right\|_{2, \Omega} \leq C_{\Omega}\|(D+k) u\|_{2, \Omega},
$$

all $k \in \Omega^{*}$. Here, $u_{\Omega}=|\Omega|^{-1} \int_{\Omega} u(x) d x$ and $C_{\Omega}=r_{\Lambda}^{-1}$.

Another operator that is closely related to lattices is the Gelfand transform $\mathcal{G}: L_{2}\left(\mathbb{R}^{d}\right) \rightarrow L_{2}\left(\Omega^{*} \times \Omega\right)$ given by

$$
(\mathcal{G} u)(k, x)=\left|\Omega^{*}\right|^{-1 / 2} \sum_{\lambda \in \Lambda} u(x+\lambda) e^{-i\langle x+\lambda, k\rangle},
$$

the series converging in $L_{2}\left(\Omega^{*} \times \Omega\right)$. It is well known that $\mathcal{G}$ is an isometric isomorphism of $L_{2}\left(\mathbb{R}^{d}\right)$ onto $L_{2}\left(\Omega^{*} \times \Omega\right)$ and an isomorphism of $H^{1}\left(\mathbb{R}^{d}\right)$ onto $L_{2}\left(\Omega^{*} ; \widetilde{H}^{1}(\Omega)\right.$ ). By duality, the Gelfand transform extends to $H^{1}\left(\mathbb{R}^{d}\right)^{*}$, so $\mathcal{G}: H^{1}\left(\mathbb{R}^{d}\right)^{*} \rightarrow L_{2}\left(\Omega^{*} ; \widetilde{H}^{1}(\Omega)\right)^{*}$.

2.2. Problem formulation. We fix a positive integer $d_{1}$ and a non-negative integer $d_{2}$; the first will be the number of the periodic directions, and the second will be the number of the non-periodic directions. We suppose for specificity that $d_{2}$ is positive; the case $d_{2}=0$ is similar, with obvious changes. Let $d=d_{1}+d_{2}$. Set $\Xi=\mathbb{R}^{d_{1}} \times \mathbb{T}_{L}^{d_{2}}, L>0$, where $\mathbb{T}_{L}^{d_{2}}$ stands for the $d_{2}$-dimensional flat torus $\mathbb{R}^{d_{2}} /$ $\left(L \mathbb{Z}^{d_{2}}\right)$; that is, a cube in $\mathbb{R}^{d_{2}}$ with opposite sides identified. Now for each $x \in \Xi$, we have $x=\left(x_{1}, x_{2}\right)$, where $x_{1} \in \mathbb{R}^{d_{1}}$ and $x_{2} \in \mathbb{T}_{L}^{d_{2}}$. The $m$ th coordinate of $x_{1}$ and the $n$th coordinate of $x_{2}$ are denoted by $x_{1, m}$ and $x_{2, n}$, respectively.

Let $\Lambda$ be a lattice in $\mathbb{R}^{d_{1}}$ acting on $\Xi$. If we denote a basic cell of $\Lambda$ by $\Omega_{1}$ and the torus $\mathbb{T}_{L}^{d_{2}}$ by $\Omega_{2}$, then $\Omega=\Omega_{1} \times \Omega_{2}$ is a fundamental domain for $\Lambda$, and $\left\{\Omega_{\lambda}\right\}_{\lambda \in \Lambda}$, where $\Omega_{\lambda}=\lambda+\Omega$, is a tiling of $\Xi$.

We now introduce a class of allowed coefficients. Let $U$ and $V$ be complex Sobolev spaces over the interior of $\Omega$ or subspaces of such spaces. We define $\mathbf{S}(U, V)$ to be the set of all complex-valued generalized functions $\gamma \in C_{0}^{\infty}(\Xi)^{*}$ such that (1) $\gamma$ is periodic with respect to $\Lambda$, (2) $\gamma \in \mathbf{M}(U, V)$, and (3) $D_{x_{2}} \gamma \in \mathbf{M}(U, V)^{d_{2}}$. We shall write $\mathbf{S}(U)$ in place of $\mathbf{S}(U, U)$.

Let $A$ be a matrix-valued function in $\mathbf{S}\left(L_{2}(\Omega)\right)^{d \times d}$ with $\operatorname{Re} A$ uniformly positive definite, $a_{1}$ and $a_{2}$ be vector-valued functions in $\mathbf{S}\left(H^{1}(\Omega), L_{2}(\Omega)\right)^{d}$, and $q$ be a complex-valued distribution in $\mathbf{S}\left(H^{1}(\Omega), H^{1}(\Omega)^{*}\right)$. Assume also that

$$
\left\|a_{1}\right\|_{\mathbf{M}}+\left\|a_{2}\right\|_{\mathbf{M}}+\|q\|_{\mathbf{M}}<\left\|(\operatorname{Re} A)^{-1}\right\|_{\mathbf{M}}^{-1} \text {. }
$$


This last requirement is not as restrictive as it might seem to be. It will turn out that the hypothesis $(6)$ is, in a sense, a weaker property than the relative $-\Delta$-formboundedness of $a_{1}^{*} D+D^{*} a_{2}+q$ with relative bound zero, so that (6) is satisfied in most cases - see Remark 2 below.

Remark 1. Our intention is to replace the scale of Lebesgue spaces with that of multiplier spaces, which prove to be perfectly suited to the problem in question. In particular, for this reason we use $\mathbf{M}\left(L_{2}(\Omega)\right)$ for the space $L_{\infty}(\Omega)$.

Let $i D_{1}$ be the vector of first partial derivatives with respect to $x_{1}$ and $i D_{2}$, with respect to $x_{2}$. Let $\mathcal{D}_{1}=\left(\begin{array}{c}D_{1} \\ 0\end{array}\right)$ and $\mathcal{D}_{2}=\left(\begin{array}{c}0 \\ D_{2}\end{array}\right)$. We shall use $\mathcal{D}$ to denote $\mathcal{D}_{1}+\mathcal{D}_{2}$. Given $\varepsilon \in \mathscr{E}=(0,1]$, we introduce the notation $\gamma^{\varepsilon}=\left(\mathcal{S}^{1 / \varepsilon} \otimes \mathcal{I}\right) \gamma\left(\mathcal{S}^{\varepsilon} \otimes \mathcal{I}\right)$ for any Sobolev multiplier $\gamma$ (if $\gamma$ is a function, then $\gamma^{\varepsilon}(x)=\gamma\left(\varepsilon^{-1} x_{1}, x_{2}\right)$ for $x \in \Xi$ ) and define the form $a^{\varepsilon}$ on $H^{1}(\Xi)$ by

$$
a^{\varepsilon}[u]=\left(A^{\varepsilon} \mathcal{D} u, \mathcal{D} u\right)_{2, \Xi}+\left(\mathcal{D} u, a_{1}^{\varepsilon} u\right)_{2, \Xi}+\left(a_{2}^{\varepsilon} u, \mathcal{D} u\right)_{2, \Xi}+\left(q^{\varepsilon} u, u\right)_{2, \Xi} .
$$

Notice that $\gamma \mapsto \gamma^{\varepsilon}$ is a bounded map of $\mathbf{M}\left(H^{m}(\Omega), L_{2}(\Omega)\right)$ onto $\mathbf{M}\left(H^{m}\left(\Omega^{\varepsilon}\right), L_{2}\left(\Omega^{\varepsilon}\right)\right)$ and of $\mathbf{M}\left(H^{m}(\Omega), H^{1}(\Omega)^{*}\right)$ onto $\mathbf{M}\left(H^{m}\left(\Omega^{\varepsilon}\right), H^{1}\left(\Omega^{\varepsilon}\right)^{*}\right)$, with norms not exceeding 1. Here $\Omega^{\varepsilon}=\varepsilon \Omega_{1} \times \Omega_{2}$. Then, since $A^{\varepsilon}, a_{n}^{\varepsilon}, n \in[2]$, and $q^{\varepsilon}$ are periodic with respect to $\varepsilon \Lambda$ and since $\left\{\left(\Omega_{\lambda}\right)^{\varepsilon}\right\}_{\lambda \in \Lambda}$ is a tiling of $\Xi$, we see that $A^{\varepsilon} \in \mathbf{M}\left(L_{2}(\Xi)\right)^{d \times d}$, $a_{n}^{\varepsilon} \in \mathbf{M}\left(H^{1}(\Xi), L_{2}(\Xi)\right)^{d}$ and $q^{\varepsilon} \in \mathbf{M}\left(H^{1}(\Xi), H^{1}(\Xi)^{*}\right)$. Furthermore, the corresponding norms are majorized by the multiplier norms of $A, a_{n}$ and $q$, respectively. Now it is clear that $\boldsymbol{a}^{\varepsilon}$ is bounded,

$$
\left|a^{\varepsilon}[u, v]\right| \leq C_{b}\|u\|_{1,2, \Xi}\|v\|_{1,2, \Xi}, \quad u, v \in H^{1}(\Xi),
$$

where

$$
C_{\mathrm{b}}=\|A\|_{\mathbf{M}}+\left\|a_{1}\right\|_{\mathbf{M}}+\left\|a_{2}\right\|_{\mathbf{M}}+\|q\|_{\mathbf{M}}
$$

Observe also that

$$
\operatorname{Re} \boldsymbol{a}^{\varepsilon}[u] \geq c_{*}\|\mathcal{D} u\|_{2, \Xi}^{2}-c_{\natural}\|u\|_{2, \Xi}^{2}, \quad u \in H^{1}(\Xi),
$$

where

$$
\begin{aligned}
& c_{*}=\left\|(\operatorname{Re} A)^{-1}\right\|_{\mathbf{M}}^{-1}-\left\|a_{1}\right\|_{\mathbf{M}}-\left\|a_{2}\right\|_{\mathbf{M}}-\|q\|_{\mathbf{M}}, \\
& c_{\natural}=2^{-1}\left(\left\|a_{1}\right\|_{\mathbf{M}}+\left\|a_{2}\right\|_{\mathbf{M}}\right)+\|q\|_{\mathbf{M}} .
\end{aligned}
$$

Since $c_{*}$ is positive, it follows that $\boldsymbol{a}^{\varepsilon}$ is coercive.

Thus, $\boldsymbol{a}^{\varepsilon}$ is strictly $m$-sectorial, with sector

$$
\mathcal{S}_{1}=\left\{z \in \mathbb{C}:|\operatorname{Im} z| \leq c_{*}^{-1} C_{b}\left(\operatorname{Re} z+c_{*}+c_{\natural}\right)\right\} .
$$

Let $\mathcal{A}_{\mu}^{\varepsilon}: H^{1}(\Xi) \rightarrow H^{1}(\Xi)^{*}$ be the operator associated with the form $a_{\mu}^{\varepsilon}=a^{\varepsilon}-\mu$. Then $\mathcal{A}_{\mu}^{\varepsilon}$ is an isomorphism whenever $\mu \notin \mathcal{S}_{1}$.

Remark 2. The hypothesis (6) is needed in order for the form $a^{\varepsilon}$ to be coercive. In fact, it can be weakened to allow those $a_{n}, n \in[2]$, and $q$ that satisfy, for any $u \in H^{1}(\Omega)$,

$$
\begin{aligned}
\left\|a_{n} u\right\|_{2, \Omega}^{2} & \leq c_{a_{n}}\|D u\|_{2, \Omega}^{2}+C_{a_{n}}\|u\|_{2, \Omega}^{2} \\
\left|(q u, u)_{2, \Omega}\right| & \leq c_{q}\|D u\|_{2, \Omega}^{2}+C_{q}\|u\|_{2, \Omega}^{2}
\end{aligned}
$$

with

$$
c_{a_{1}}^{1 / 2}+c_{a_{2}}^{1 / 2}+c_{q}<\left\|(\operatorname{Re} A)^{-1}\right\|_{\mathbf{M}}^{-1} .
$$


Indeed, since we are interested in estimating operator norms (see Theorems 13 ) and since $\mathcal{S}^{\delta}$ is an isomorphism, we may replace $\mathcal{A}^{\varepsilon}$ by $\hat{\mathcal{A}}^{\varepsilon}=\mathcal{S}^{\delta} \mathcal{A}^{\varepsilon}\left(\mathcal{S}^{\delta}\right)^{-1}$. (Here, we realize the torus $\Omega_{2}=\mathbb{T}_{L}^{d_{2}}$ as the cube $[0, L]^{d_{2}}$ with opposite sides identified, and, in this sense, $\delta^{-1} \mathbb{T}_{L}^{d_{2}}$ is well defined and equals $\left.\mathbb{T}_{\delta^{-1} L}^{d_{2}}.\right)$ It is easy to see that the coefficients of $\hat{\mathcal{A}}^{1}$ are given by $\hat{A}=\delta^{-2} \mathcal{S}^{\delta} A \mathcal{S}^{1 / \delta}, \hat{a}_{n}=\delta^{-1} \mathcal{S}^{\delta} a_{n} \mathcal{S}^{1 / \delta}$ and $\hat{q}=$ $\mathcal{S}^{\delta} q \mathcal{S}^{1 / \delta}$. Therefore, if we take $\delta$ so that $\delta^{2} \leq \min \left\{c_{a_{1}} C_{a_{1}}^{-1}, c_{a_{2}} C_{a_{2}}^{-1}, c_{q} C_{q}^{-1}\right\}$, then

$$
\begin{aligned}
\left\|\hat{a}_{1}\right\|_{\mathbf{M}}+\left\|\hat{a}_{2}\right\|_{\mathbf{M}}+\|\hat{q}\|_{\mathbf{M}} & \leq \delta^{-2}\left(c_{a_{1}}^{1 / 2}+c_{a_{2}}^{1 / 2}+c_{q}\right) \\
& <\delta^{-2}\left\|(\operatorname{Re} A)^{-1}\right\|_{\mathbf{M}}^{-1}=\left\|(\operatorname{Re} \hat{A})^{-1}\right\|_{\mathbf{M}}^{-1} ;
\end{aligned}
$$

that is, the hypothesis $(6)$ holds for $\hat{\mathcal{A}}^{\varepsilon}$. We note that the class of operators such as $\mathcal{A}^{\varepsilon}$ here is broad enough to cover most cases that arise in applications - see an example below.

We are interested in approximations for $\left(\mathcal{A}_{\mu}^{\varepsilon}\right)^{-1}$ and $\mathcal{D}\left(\mathcal{A}_{\mu}^{\varepsilon}\right)^{-1}$ in the operator norm on $L_{2}(\Xi)$. In order to describe these approximations, we define the effective operator and two different correctors.

2.3. Effective operator. Let $N$ be the weak solution of

$$
\mathcal{D}_{1}^{*} A\left(\mathcal{D}_{1} N+I\right)=0
$$

in $L_{2}\left(\Omega_{2} ; \widetilde{H}_{0}^{1}\left(\Omega_{1}\right)\right)^{1 \times d}$, and $M$ be the weak solution of

$$
\mathcal{D}_{1}^{*}\left(A \mathcal{D}_{1} M+a_{2}\right)=0
$$

in $L_{2}\left(\Omega_{2} ; \widetilde{H}_{0}^{1}\left(\Omega_{1}\right)\right)$. We know that $N$ and $M$ exist and are unique, since we may rewrite these problems as

$$
D_{1}^{*} A_{11} D_{1} u=D_{1}^{*} f
$$

with an $f$ in $L_{2}(\Omega)^{d_{1}}$ and $u \in L_{2}\left(\Omega_{2} ; \widetilde{H}_{0}^{1}\left(\Omega_{1}\right)\right)$ to be found. Notice in passing that such a $u$ satisfies

$$
\left(A_{11}\left(\cdot, x_{2}\right) D_{1} u\left(\cdot, x_{2}\right)-f\left(\cdot, x_{2}\right), D_{1} v\right)_{2, \Omega_{1}}=0
$$

for almost every $x_{2} \in \Omega_{2}$ and all $v \in \widetilde{H}^{1}\left(\Omega_{1}\right)$.

We now provide some elementary properties of $N$ and $M$ (cf. SU2, Proposition 8.2]).

Lemma 1. Let $u$ be the weak solution of the problem (17) where the function $f$ is in $\mathbf{M}\left(H^{m}(\Omega) ; L_{2}(\Omega)\right)^{d_{1}}, m$ a non-negative integer. Then $D_{1} u \in \mathbf{M}\left(H^{m}\left(\Omega_{2}\right), L_{2}(\Omega)\right)^{d_{1}}$ and

$$
\left\|D_{1} u\right\|_{\mathbf{M}} \leq\left|\Omega_{1}\right|^{1 / 2}\left\|(\operatorname{Re} A)^{-1}\right\|_{\mathbf{M}}\|f\|_{\mathbf{M}} .
$$

If, in addition, $D_{2, n} f \in \mathbf{M}\left(H^{m}(\Omega) ; L_{2}(\Omega)\right)^{d_{1}}$ for some $n \in\left[d_{2}\right]$, then $D_{2, n} D_{1} u \in$ $\mathbf{M}\left(H^{m}\left(\Omega_{2}\right), L_{2}(\Omega)\right)^{d_{1}}$ and

$\left\|D_{2, n} D_{1} u\right\|_{\mathbf{M}} \leq\left|\Omega_{1}\right|^{1 / 2}\left\|(\operatorname{Re} A)^{-1}\right\|_{\mathbf{M}}\left(\left\|D_{2, n} A\right\|_{\mathbf{M}}\left\|(\operatorname{Re} A)^{-1}\right\|_{\mathbf{M}}\|f\|_{\mathbf{M}}+\left\|D_{2, n} f\right\|_{\mathbf{M}}\right)$.

Proof. Let $v=u|w|^{2}$ with $w \in C^{m}\left(\Omega_{2}\right)$. Then $v \in L_{2}\left(\Omega_{2} ; \widetilde{H}_{0}^{1}\left(\Omega_{1}\right)\right)$, and we can apply both sides of (17) to $v$, obtaining

$$
\left\|\left(D_{1} u\right) w\right\|_{2, \Omega} \leq\left\|\left(\operatorname{Re} A_{11}\right)^{-1}\right\|_{\mathbf{M}}\|f w\|_{2, \Omega} .
$$

This proves the first assertion. 
Suppose now $D_{2, n} f \in \mathbf{M}\left(H^{m}(\Omega) ; L_{2}(\Omega)\right)^{d_{1}}$. We know that $D_{2, n} f \in L_{2}(\Omega)^{d_{1}}$, so $D_{2, n} u$ exists and belongs to $L_{2}\left(\Omega_{2} ; \widetilde{H}_{0}^{1}\left(\Omega_{1}\right)\right)$, which may be verified by using the difference quotient technique of NIRENBERG. Therefore, we can write

$$
D_{1}^{*} A_{11} D_{1} D_{2, n} u=D_{1}^{*}\left(D_{2, n} f-\left(D_{2, n} A_{11}\right) D_{1} u\right) .
$$

Applying both sides of the last equality to $v=\left(D_{2, n} u\right)|w|^{2}$ with $w \in C^{m}\left(\Omega_{2}\right)$ yields

$$
\left\|\left(D_{1} D_{2, n} u\right) w\right\|_{2, \Omega} \leq\left\|\left(\operatorname{Re} A_{11}\right)^{-1}\right\|_{\mathbf{M}}\left(\left\|\left(D_{2, n} A_{11}\right)\left(D_{1} u\right) w\right\|_{2, \Omega}+\left\|\left(D_{2, n} f\right) w\right\|_{2, \Omega}\right),
$$

and the second assertion follows.

From the above lemma and the Poincaré inequality (5), we conclude that $N \in$ $\mathbf{S}\left(L_{2}\left(\Omega_{2}\right), L_{2}(\Omega)\right)^{1 \times d}$ and $\mathcal{D}_{1} N \in \mathbf{S}\left(L_{2}\left(\Omega_{2}\right), L_{2}(\Omega)\right)^{d \times d}$, while $M \in \mathbf{S}\left(H^{1}\left(\Omega_{2}\right), L_{2}(\Omega)\right)$ and $\mathcal{D}_{1} M \in \mathbf{S}\left(H^{1}\left(\Omega_{2}\right), L_{2}(\Omega)\right)^{d}$.

We now turn to the effective coefficients.

Let

$$
A^{0}=\left|\Omega_{1}\right|^{-1} \int_{\Omega_{1}} A\left(\mathcal{D}_{1} N+I\right) d y_{1}
$$

Then, from the properties of $A$ and $N$, we have $A^{0} \in \mathbf{S}\left(L_{2}\left(\Omega_{2}\right)\right)^{d \times d}$. It is a standard fact (see [ZHKO, Section 1.6]) that if $\operatorname{Re} A$ is positive definite, then

$$
\operatorname{Re} A^{0} \geq\left(\left|\Omega_{1}\right|^{-1} \int_{\Omega_{1}}(\operatorname{Re} A)^{-1} d y_{1}\right)^{-1}
$$

This implies that $\operatorname{Re} A^{0}$ is also positive definite and furthermore $\left(\operatorname{Re} A^{0}\right)^{-1}$ is in $\mathbf{M}\left(L_{2}\left(\Omega_{2}\right)\right)^{d \times d}$ and $\left\|\left(\operatorname{Re} A^{0}\right)^{-1}\right\|_{\mathbf{M}} \leq\left\|(\operatorname{Re} A)^{-1}\right\|_{\mathbf{M}}$.

Next, we define the functions

$$
\begin{aligned}
& a_{1}^{0}=\left|\Omega_{1}\right|^{-1} \int_{\Omega_{1}}\left(\mathcal{D}_{1} N+I\right)^{*} a_{1} d y_{1}, \\
& a_{2}^{0}=\left|\Omega_{1}\right|^{-1} \int_{\Omega_{1}}\left(A \mathcal{D}_{1} M+a_{2}\right) d y_{1} .
\end{aligned}
$$

Both of these are in $\mathbf{S}\left(H^{1}\left(\Omega_{2}\right), L_{2}\left(\Omega_{2}\right)\right)^{d}$, as can be seen from the properties of $A$, $a_{1}, a_{2}$ and $N, M$.

Finally, let $q^{0}$ correspond to the form

$$
\left(q^{0} u, u\right)_{2, \Omega_{2}}=\left|\Omega_{1}\right|^{-1}(q u, u)_{2, \Omega}+\left|\Omega_{1}\right|^{-1}\left(a_{1}^{*} \mathcal{D}_{1} M u, u\right)_{2, \Omega}
$$

on $H^{1}\left(\Omega_{2}\right)$. By the properties of $a_{1}, q$ and $M$, we obtain $q^{0} \in \mathbf{S}\left(H^{1}\left(\Omega_{2}\right), H^{1}\left(\Omega_{2}\right)^{*}\right)$. Notice that, in the case when $q$ is a function, we have, as usual,

$$
q^{0}=\left|\Omega_{1}\right|^{-1} \int_{\Omega_{1}} q d y_{1}+\left|\Omega_{1}\right|^{-1} \int_{\Omega_{1}} a_{1}^{*} \mathcal{D}_{1} M d y_{1} .
$$

We are almost ready to define the effective operator. Consider the form $a^{0}$ on $H^{1}(\Xi)$ given by

$$
a^{0}[u]=\left(A^{0} \mathcal{D} u, \mathcal{D} u\right)_{2, \Xi}+\left(\mathcal{D} u, a_{1}^{0} u\right)_{2, \Xi}+\left(a_{2}^{0} u, \mathcal{D} u\right)_{2, \Xi}+\left(q^{0} u, u\right)_{2, \Xi}
$$

where

$$
\left(q^{0} u, u\right)_{2, \Xi}=\int_{\mathbb{R}^{d_{1}}}\left(q^{0} u\left(x_{1}, \cdot\right), u\left(x_{1}, \cdot\right)\right)_{2, \Omega_{2}} d x_{1} .
$$

Then $a^{0}$ is plainly bounded,

$$
\left|a^{0}[u, v]\right| \leq C_{b}^{0}\|u\|_{1,2, \Xi}\|v\|_{1,2, \Xi}, \quad u, v \in H^{1}(\Xi),
$$


with

$$
C_{\mathrm{b}}^{0}=\left\|A^{0}\right\|_{\mathbf{M}}+\left\|a_{1}^{0}\right\|_{\mathbf{M}}+\left\|a_{2}^{0}\right\|_{\mathbf{M}}+\left\|q^{0}\right\|_{\mathbf{M}}
$$

In a moment, we shall see that it is coercive.

Lemma 2. Let $\check{a}^{0}$ be the form on $H^{1}(\Xi) \oplus L_{2}\left(\Xi ; \widetilde{H}_{0}^{1}\left(\Omega_{1}\right)\right)$ given by

$$
\begin{aligned}
& \check{a}^{0}[\check{u}]=\left|\Omega_{1}\right|^{-1} \int_{\Xi} \int_{\Omega_{1}}(\left\langle A\left(y_{1}, x_{2}\right) \check{\mathcal{D}} \check{u}\left(x, y_{1}\right), \check{\mathcal{D}} \check{u}\left(x, y_{1}\right)\right\rangle \\
&+\left\langle\check{\mathcal{D}} \check{u}\left(x, y_{1}\right), a_{1}\left(y_{1}, x_{2}\right) \check{u}_{1}(x)\right\rangle \\
&\left.+\left\langle a_{2}\left(y_{1}, x_{2}\right) \check{u}_{1}(x), \check{\mathcal{D}} \check{u}\left(x, y_{1}\right)\right\rangle\right) d x d y_{1} \\
&+\left|\Omega_{1}\right|^{-1} \int_{\mathbb{R}^{d_{1}}}\left(q \check{u}_{1}\left(x_{1}, \cdot\right), \check{u}_{1}\left(x_{1}, \cdot\right)\right)_{2, \Omega} d x_{1}
\end{aligned}
$$

where $\check{u}=\left(\check{u}_{1}, \check{u}_{2}\right)$ and $\check{\mathcal{D}} \check{u}\left(x, y_{1}\right)=\mathcal{D}_{x} \check{u}_{1}(x)+\mathcal{D}_{y_{1}} \check{u}_{2}\left(x, y_{1}\right)$. Then $\check{a}^{0}$ is coercive and

$$
\operatorname{Re} \check{\boldsymbol{a}}^{0}[\check{u}] \geq c_{*}\left|\Omega_{1}\right|^{-1}\|\check{\mathcal{D}} \check{u}\|_{2, \Xi \times \Omega_{1}}^{2}-c_{\natural}\left\|\check{u}_{1}\right\|_{2, \Xi}^{2},
$$

all $\check{u} \in H^{1}(\Xi) \oplus L_{2}\left(\Xi ; \widetilde{H}_{0}^{1}\left(\Omega_{1}\right)\right)$.

Proof. While the proof is quite similar to that of $(9)$, there is a difference: the variables $x_{1}$ and $y_{1}$ in the definition of $\check{a}^{0}$ are "mixed", so that we cannot treat the lower-order terms as before.

We begin with a first-order term. By Cauchy's inequality, we have

$$
\begin{aligned}
& \left|\int_{\Xi} \int_{\Omega_{1}}\left\langle\check{\mathcal{D}} \check{u}\left(x, y_{1}\right), a_{1}\left(y_{1}, x_{2}\right) \check{u}_{1}(x)\right\rangle d x d y_{1}\right| \\
& \quad \leq\|\check{\mathcal{D}} \check{u}\|_{2, \Xi \times \Omega_{1}}\left(\int_{\Xi} \int_{\Omega_{1}}\left|a_{1}\left(y_{1}, x_{2}\right) \check{u}_{1}(x)\right|^{2} d x d y_{1}\right)^{1 / 2} .
\end{aligned}
$$

Let $v$ denote the mapping $y \mapsto\left(\int_{\mathbb{R}^{d_{1}}}\left|\check{u}_{1}\left(x_{1}, y_{2}\right)\right|^{2} d x_{1}\right)^{1 / 2}$. Then $v \in H^{1}(\Omega)$, with $\|v\|_{1,2, \Omega} \leq\left|\Omega_{1}\right|^{1 / 2}\left\|\check{u}_{1}\right\|_{1,2, \Xi}$, and

$$
\int_{\Xi} \int_{\Omega_{1}}\left|a_{1}\left(y_{1}, x_{2}\right) \check{u}_{1}(x)\right|^{2} d x d y_{1}=\left\|a_{1} v\right\|_{2, \Omega}^{2} .
$$

As a result,

We have used here the fact that, by Stokes' theorem,

$$
\begin{aligned}
& \left|\int_{\Xi} \int_{\Omega_{1}}\left\langle\check{\mathcal{D}} \check{u}\left(x, y_{1}\right), a_{1}\left(y_{1}, x_{2}\right) \check{u}_{1}(x)\right\rangle d x d y_{1}\right| \\
& \quad \leq\left|\Omega_{1}\right|^{1 / 2}\left\|a_{1}\right\|_{\mathbf{M}}\|\check{\mathcal{D}} \check{u}\|_{2, \Xi \times \Omega_{1}}\left\|\check{u}_{1}\right\|_{1,2, \Xi} \\
& \quad \leq\left\|a_{1}\right\|_{\mathbf{M}}\left(\|\check{\mathcal{D}} \check{u}\|_{2, \Xi \times \Omega_{1}}^{2}+2^{-1}\left|\Omega_{1}\right|\left\|\check{u}_{1}\right\|_{2, \Xi}^{2}\right) .
\end{aligned}
$$

$$
\|\check{\mathcal{D}} \check{u}\|_{2, \Xi \times \Omega_{1}}^{2}=\left|\Omega_{1}\right|\left\|\mathcal{D}_{x} \check{u}_{1}\right\|_{2, \Xi}^{2}+\left\|\mathcal{D}_{y_{1}} \check{u}_{2}\right\|_{2, \Xi \times \Omega_{1}}^{2} .
$$

We may likewise prove that

$$
\begin{aligned}
& \left|\int_{\Xi} \int_{\Omega_{1}}\left\langle a_{2}\left(y_{1}, x_{2}\right) \check{u}_{1}(x), \check{\mathcal{D}} \check{u}\left(x, y_{1}\right)\right\rangle d x d y_{1}\right| \\
& \quad \leq\left\|a_{2}\right\|_{\mathbf{M}}\left(\|\check{\mathcal{D}} \check{u}\|_{2, \Xi \times \Omega_{1}}^{2}+2^{-1}\left|\Omega_{1}\right|\left\|\check{u}_{1}\right\|_{2, \Xi}^{2}\right)
\end{aligned}
$$

and

$$
\begin{aligned}
& \left|\int_{\mathbb{R}^{d_{1}}}\left(q \check{u}_{1}\left(x_{1}, \cdot\right), \check{u}_{1}\left(x_{1}, \cdot\right)\right)_{2, \Omega} d x_{1}\right| \\
& \quad=\left|(q v, v)_{2, \Omega}\right| \leq\|q\|_{\mathbf{M}}\left(\|\check{\mathcal{D}} \check{u}\|_{2, \Xi \times \Omega_{1}}^{2}+\left|\Omega_{1}\right|\left\|\check{u}_{1}\right\|_{2, \Xi}^{2}\right) .
\end{aligned}
$$


Combining these inequalities with

$$
\begin{aligned}
& \operatorname{Re} \int_{\Xi} \int_{\Omega_{1}}\left\langle A\left(y_{1}, x_{2}\right) \check{\mathcal{D}} \check{u}\left(x, y_{1}\right), \check{\mathcal{D}} \check{u}\left(x, y_{1}\right)\right\rangle d x d y_{1} \\
& \quad \geq\left\|(\operatorname{Re} A)^{-1}\right\|_{\mathbf{M}}^{-1}\|\check{\mathcal{D}} \check{u}\|_{2, \Xi \times \Omega_{1}}^{2},
\end{aligned}
$$

which is obvious, gives 26.

Remark 3. The form $\check{a}^{0}$ is associated with the two-scale homogenized system, first proposed by Allaire $\mathrm{AL}$ in the context of two-scale convergence. See also [LNW] for a self-contained approach to this matter.

Now we wish to relate the form $\check{a}^{0}$ to $a^{0}$. Fix a $u \in H^{1}(\Xi)$. Let $\check{u}_{1}(x)=$ $u(x)$ and $\check{u}_{2}\left(x, y_{1}\right)=N\left(y_{1}, x_{2}\right) \mathcal{D}_{x} u(x)+M\left(y_{1}, x_{2}\right) u(x)$. We claim that $\check{u}$ belongs to $H^{1}(\Xi) \oplus L_{2}\left(\Xi ; \widetilde{H}_{0}^{1}\left(\Omega_{1}\right)\right)$. Indeed, $\check{u}_{2}\left(x, y_{1}\right)$ has a derivative with respect to $y_{1}$, and, by reasoning explained in the proof of Lemma 2 , it lies in $L_{2}\left(\Xi \times \Omega_{1}\right)^{d_{1}}$ (notice here that $\mathcal{D}_{1} N$ and $\mathcal{D}_{1} M$ are multipliers). Applying identities for $N$ and $M$ in the form 18), we find that $\check{a}^{0}[\check{u}, \check{v}]=0$ for all $\check{v} \in\{0\} \oplus L_{2}\left(\Xi ; \widetilde{H}_{0}^{1}\left(\Omega_{1}\right)\right)$, and therefore $\check{\boldsymbol{a}}^{0}[\check{u}]=\breve{a}^{0}[\check{u}, u \oplus 0]$. Now it follows from the definitions of the effective coefficients that

$$
\check{a}^{0}[\check{u}]=a^{0}[u]
$$

for every $u \in H^{1}(\Xi)$, which is the desired relation.

Since $\check{\mathfrak{a}}^{0}$ is coercive, the above identity tells us that so is $\boldsymbol{a}^{0}$, with

$$
\operatorname{Re} a^{0}[u] \geq c_{*}\|\mathcal{D} u\|_{2, \Xi}^{2}-c_{\natural}\|u\|_{2, \Xi}^{2}, \quad u \in H^{1}(\Xi) .
$$

Hence, the form $a^{0}$ is strictly $m$-sectorial, with sector

$$
\mathcal{S}_{0}=\left\{z \in \mathbb{C}:|\operatorname{Im} z| \leq c_{*}^{-1} C_{b}^{0}\left(\operatorname{Re} z+c_{*}+c_{\natural}\right)\right\} .
$$

Corresponding to $a_{\mu}^{0}=a^{0}-\mu$ there is an operator $\mathcal{A}_{\mu}^{0}=\mathcal{A}^{0}-\mu: H^{1}(\Xi) \rightarrow H^{1}(\Xi)^{*}$, which is an isomorphism provided that $\mu \notin \delta_{0}$. For such a $\mu,\left(\mathcal{A}_{\mu}^{0}\right)^{-1}$ maps $L_{2}(\Xi)$ onto $H^{2}(\Xi)$. (This can be shown by using the difference quotient technique of NirenberG; see the proof of Lemma 4 for further details on this matter.) We denote the largest of the sectors $\delta_{0}$ and $\delta_{1}$ by $\delta$.

2.4. Correctors. We introduce two types of correctors. The first, denoted $\mathcal{K}_{\mu}^{\varepsilon}$, will be needed to obtain the approximation for $\mathcal{D}_{1}\left(\mathcal{A}_{\mu}^{\varepsilon}\right)^{-1}$ and is defined as follows. Let $\mathcal{P}^{\varepsilon}$ be the pseudodifferential operator in the $x_{1}$-variable with symbol $\chi_{\varepsilon^{-1}} \Omega_{1}^{*}$, where $\chi_{\varepsilon^{-1} \Omega_{1}^{*}}$ is the characteristic function of the set $\varepsilon^{-1} \Omega_{1}^{*}$, or, to put it differently,

$$
\mathcal{P}^{\varepsilon}=(\mathcal{F} \otimes \mathcal{I})^{*} \chi_{\varepsilon^{-1} \Omega_{1}^{*}}(\mathcal{F} \otimes \mathcal{I}) .
$$

Here $\mathcal{F}$ is the Fourier transform in $L_{2}\left(\mathbb{R}^{d_{1}}\right)$. Then the corrector $\mathcal{K}_{\mu}^{\varepsilon}: L_{2}(\Xi) \rightarrow$ $H^{1}(\Xi)$ for $\mathcal{A}^{\varepsilon}$ is given by

$$
\mathcal{K}_{\mu}^{\varepsilon}=\left(N^{\varepsilon} \mathcal{D}+M^{\varepsilon}\right)\left(\mathcal{A}_{\mu}^{0}\right)^{-1} \mathcal{P}^{\varepsilon} .
$$

We remark that, while $\left(N^{\varepsilon} \mathcal{D}+M^{\varepsilon}\right)\left(\mathcal{A}_{\mu}^{0}\right)^{-1} f$, with $f \in L_{2}(\Xi)$, is not generally in $H^{1}(\Xi)$ (not even in $L_{2}(\Xi)$ ), the function $\mathcal{K}_{\mu}^{\varepsilon} f$ always is, which may be proved by applying the scaling transformation and the Gelfand transform (see (55)) and then using the properties of $N$ and $M$ (see Lemma 5). What is more, these calculations show that $\mathcal{K}_{\mu}^{\varepsilon}$ is a bounded operator. 
The second corrector, denoted $\mathcal{C}_{\mu}^{\varepsilon}$, will be needed for a more subtle result. If $k$ is a vector in $\mathbb{R}^{d_{1}}$ and $\mathcal{K}$ is the corresponding element of $\mathbb{R}^{d_{1}} \oplus\{0\}$, then we define differential expressions

$\mathcal{S}\left(k ; y_{1}\right)=\left(\left(\mathcal{K}+\mathcal{D}_{2}\right)^{*} A\left(y_{1}, \cdot\right)+a_{1}^{*}\left(y_{1}, \cdot\right)\right)\left(\mathcal{K}+\mathcal{D}_{2}\right)+\left(\mathcal{K}+\mathcal{D}_{2}\right)^{*} a_{2}\left(y_{1}, \cdot\right)+q\left(y_{1}, \cdot\right)$, $\mathcal{T}\left(k ; y_{1}\right)=\left(\left(\kappa+\mathcal{D}_{2}\right)^{*} A\left(y_{1}, \cdot\right)+a_{1}^{*}\left(y_{1}, \cdot\right)\right) \mathcal{D}_{y_{1}}$

and families of operators

$$
\begin{aligned}
\mathcal{A}_{\mu}^{0}(k) & =\left(\mathcal{K}+\mathcal{D}_{2}\right)^{*} A^{0}\left(\mathcal{K}+\mathcal{D}_{2}\right)+\left(a_{1}^{0}\right)^{*}\left(\mathcal{K}+\mathcal{D}_{2}\right)+\left(\mathcal{K}+\mathcal{D}_{2}\right)^{*} a_{2}^{0}+q^{0}-\mu, \\
\mathcal{K}_{\mu}\left(k ; y_{1}\right) & =\left(N\left(y_{1}, \cdot\right)\left(\mathcal{K}+\mathcal{D}_{2}\right)+M\left(y_{1}, \cdot\right)\right)\left(\mathcal{A}_{\mu}^{0}(k)\right)^{-1} .
\end{aligned}
$$

Let $\left(\mathcal{A}_{\mu}^{\varepsilon}\right)^{+}$be the adjoint of $\mathcal{A}_{\mu}^{\varepsilon}$. For the operator $\left(\mathcal{A}_{\mu}^{\varepsilon}\right)^{+}$, we construct the effective operator $\left(\mathcal{A}_{\mu}^{0}\right)^{+}$and the corrector $\left(\mathcal{K}_{\mu}^{\varepsilon}\right)^{+}$, as well as the families $\mathcal{A}_{\mu}^{0}(k)^{+}$ and $\mathcal{K}_{\mu}\left(k ; y_{1}\right)^{+}$. (It may be noted in passing that $\left(\mathcal{A}_{\mu}^{0}\right)^{+}$is the adjoint of $\mathcal{A}_{\mu}^{0}$.) Finally, let $\mathcal{L}_{\mu}$ be the pseudodifferential operator in the $x_{1}$-variable with operatorvalued symbol $k \mapsto \mathcal{L}_{\mu}(k): L_{2}\left(\Omega_{2}\right) \rightarrow L_{2}\left(\Omega_{2}\right)$ where

$$
\mathcal{L}_{\mu}(k)=\left|\Omega_{1}\right|^{-1} \int_{\Omega_{1}}\left(\mathcal{K}_{\mu}\left(k ; y_{1}\right)^{+}\right)^{*}\left(\mathcal{S}\left(k ; y_{1}\right)\left(\mathcal{A}_{\mu}^{0}(k)\right)^{-1}+\mathcal{T}\left(k ; y_{1}\right) \mathcal{K}_{\mu}\left(k ; y_{1}\right)\right) d y_{1}
$$

that is,

$$
\mathcal{L}_{\mu}=(\mathcal{F} \otimes \mathcal{I})^{*} \mathcal{L}_{\mu}(\cdot)(\mathcal{F} \otimes \mathcal{I}) .
$$

The operator $\mathcal{L}_{\mu}^{+}$is constructed similarly. The corrector $\mathcal{C}_{\mu}^{\varepsilon}: L_{2}(\Xi) \rightarrow L_{2}(\Xi)$ is then defined by the formula

$$
\mathcal{C}_{\mu}^{\varepsilon}=\left(\mathcal{K}_{\mu}^{\varepsilon}-\mathcal{L}_{\mu}\right)+\left(\left(\mathcal{K}_{\mu}^{\varepsilon}\right)^{+}-\mathcal{L}_{\mu}^{+}\right)^{*}
$$

We will see in what follows that $\mathcal{C}_{\mu}^{\varepsilon}$ is continuous.

Remark 4. Notice that, since $\mathcal{A}_{\mu}^{0}(\cdot)$ is the symbol of $\mathcal{A}_{\mu}^{0}$ in the above indicated sense, $\mathcal{L}_{\mu}$ can be written as

$$
\mathcal{L}_{\mu}=\left(\mathcal{A}_{\mu}^{0}\right)^{-1} \mathcal{M}\left(\mathcal{A}_{\mu}^{0}\right)^{-1}
$$

where $\mathcal{M}: H^{2}(\Xi) \rightarrow H^{1}(\Xi)^{*}$ is a third-order differential operator with coefficients depending only on $x_{2}$.

We conclude this section with an example of the operator $\mathcal{A}^{\varepsilon}$.

2.5. An example. Let $d>1$ and $p>d$. From the Ehrling lemma, we know that if $\gamma \in L_{p}(\Omega)$, then $\gamma \in \mathbf{M}\left(H^{1}(\Omega), L_{2}(\Omega)\right)$ and for all $\epsilon>0$ there is a $C_{\gamma}(\epsilon)>0$, depending on $d, p, \Omega$ and $\|\gamma\|_{p, \Omega}$, such that

$$
\|\gamma u\|_{2, \Omega}^{2} \leq \epsilon\|D u\|_{2, \Omega}^{2}+C_{\gamma}(\epsilon)\|u\|_{2, \Omega}^{2}, \quad u \in H^{1}(\Omega) .
$$

As an example of a multiplier between $H^{1}(\Omega)$ and $H^{1}(\Omega)^{*}$, let $\delta_{\Sigma}$ be the Dirac distribution on a $d-1$ dimensional Lipschitz surface $\Sigma$ in $\Omega$ and let $\sigma$ be a function in $L_{p-1}(\Sigma)$. Again, for each $\epsilon>0$ there is a $C_{\sigma}(\epsilon)$, depending on $d, p, \Omega, \Sigma$ and $\|\sigma\|_{p-1, \Sigma}$, such that

$$
\left|\left(\sigma \delta_{\Sigma} u, u\right)_{2, \Omega}\right| \leq \epsilon\|D u\|_{2, \Omega}^{2}+C_{\sigma}(\epsilon)\|u\|_{2, \Omega}^{2}, \quad u \in H^{1}(\Omega) .
$$

Equipped with this information, we consider a periodic operator on $L_{2}(\Xi)$ of the form

$$
\mathcal{H}^{\varepsilon}=\left(\mathcal{D}-A_{1}^{\varepsilon}\right)^{*} g^{\varepsilon}\left(\mathcal{D}-A_{2}^{\varepsilon}\right)+V^{\varepsilon}
$$


We may think of $\mathcal{H}^{\varepsilon}$ as a (possibly non-self-adjoint) periodic Schrödinger operator with magnetic and electric potentials that is associated with metric $g^{\varepsilon}$. Suppose that $g$ is a periodic function in $\operatorname{Lip}\left(\Omega_{2} ; L_{\infty}\left(\Omega_{1}\right)\right)^{d \times d}$ and $\operatorname{Re} g$ is uniformly positive definite. Let $A_{1}$ and $A_{2}$ be periodic functions in $W_{p}^{1}\left(\Omega_{2} ; L_{p}\left(\Omega_{1}\right)\right)^{d}$. Finally, let $\Sigma$ be a $d-1$ dimensional periodic Lipschitz surface in $\Xi$. Then we assume that $V$ is the sum of a periodic function $\hat{V} \in W_{p / 2}^{1}\left(\Omega_{2} ; L_{p / 2}\left(\Omega_{1}\right)\right)$ and a distribution $\sigma \delta_{\Sigma}$ with periodic $\sigma \in W_{p-1}^{1}(\Sigma \cap \Omega)$. Clearly, $\mathcal{H}^{\varepsilon}$ thus defined can be expressed in the form

$$
\mathcal{H}^{\varepsilon}=\mathcal{D}^{*} A^{\varepsilon} \mathcal{D}+\left(a_{1}^{\varepsilon}\right)^{*} \mathcal{D}+\mathcal{D}^{*} a_{2}^{\varepsilon}+q^{\varepsilon},
$$

where the coefficients satisfy the properties $(12)-(14)$ in Remark 2 . So our result applies to $\mathcal{H}^{\varepsilon}$.

It is straightforward to construct an analogous example for the case $d=1$. Now we take $\Sigma$ to be a discrete periodic set of points in $\mathbb{R}$ and assume that $g \in L_{\infty}(\Omega)$ with $\operatorname{Re} g$ uniformly positive definite, $A_{1}, A_{2} \in L_{2}(\Omega)$ and $V=\hat{V}+\sigma \delta_{\Sigma}$ where $\hat{V}$ lies in $L_{1}(\Omega)$ and $\sigma$ is a periodic function on $\Sigma$.

We note that the potential $V^{\varepsilon}$ may also involve a singular term $\varepsilon^{-1} W^{\varepsilon}$ with a suitable function $W$. We refer the reader to [SU3, Section 11] for the details.

\section{MAin REsults}

We now state the principal results of the present paper.

Theorem 1. If $\mu \notin \mathcal{S}$, then for any $\varepsilon \in \mathscr{E}$ we have

$$
\begin{aligned}
\left\|\left(\mathcal{A}_{\mu}^{\varepsilon}\right)^{-1}-\left(\mathcal{A}_{\mu}^{0}\right)^{-1}\right\|_{\mathbf{B}\left(L_{2}(\Xi)\right)} & \lesssim \varepsilon, \\
\left\|\mathcal{D}_{2}\left(\mathcal{A}_{\mu}^{\varepsilon}\right)^{-1}-\mathcal{D}_{2}\left(\mathcal{A}_{\mu}^{0}\right)^{-1}\right\|_{\mathbf{B}\left(L_{2}(\Xi)\right)^{d}} & \lesssim \varepsilon .
\end{aligned}
$$

The estimates are sharp with respect to the order, and the constants depend only on $r_{\Lambda}, \mu$ and the multiplier norms of the coefficients.

Theorem 2. If $\mu \notin \mathcal{S}$, then for any $\varepsilon \in \mathscr{E}$ we have

$$
\left\|\mathcal{D}_{1}\left(\mathcal{A}_{\mu}^{\varepsilon}\right)^{-1}-\mathcal{D}_{1}\left(\mathcal{A}_{\mu}^{0}\right)^{-1}-\varepsilon \mathcal{D}_{1} \mathcal{K}_{\mu}^{\varepsilon}\right\|_{\mathbf{B}\left(L_{2}(\Xi)\right)^{d}} \lesssim \varepsilon .
$$

The estimate is sharp with respect to the order, and the constant depends only on $r_{\Lambda}, \mu$ and the multiplier norms of the coefficients.

Theorem 3. If $\mu \notin \mathcal{S}$, then for any $\varepsilon \in \mathscr{E}$ we have

$$
\left\|\left(\mathcal{A}_{\mu}^{\varepsilon}\right)^{-1}-\left(\mathcal{A}_{\mu}^{0}\right)^{-1}-\varepsilon \mathcal{C}_{\mu}^{\varepsilon}\right\|_{\mathbf{B}\left(L_{2}(\Xi)\right)} \lesssim \varepsilon^{2} .
$$

The estimate is sharp with respect to the order, and the constant depends only on $r_{\Lambda}, \mu$ and the multiplier norms of the coefficients.

Remark 5. Although it is possible to write down all the constants explicitly, we do not do so here. In particular, we write $\alpha \lesssim \beta$ to mean $\alpha \leq C \beta$ where $C$ is a positive constant depending only on $r_{\Lambda}, \mu$ and the multiplier norms of the coefficients.

Remark 6. Using the resolvent identity, we can transfer the estimates in Theorems $1-3$ to those $\mu \in \mathcal{S}$ for which $\mathcal{A}_{\mu}^{\varepsilon}$ (at least for each $\varepsilon$ in an interval $\left(0, \varepsilon_{\mu}\right]$ ) and $\mathcal{A}_{\mu}^{0}$, when viewed as operators on $L_{2}(\Xi)$, have bounded inverses with norms majorized by constants independent of $\varepsilon$. For instance, the estimates hold if $\mu$ is in the resolvent set of the effective operator, but in this case we have no control over $\varepsilon_{\mu}$. 
Remark 7. The hypothesis that the coefficients have weak derivatives with respect to the non-periodic variable is crucial to our analysis and reflects the fact that the roles of the two variables are quite different. Roughly speaking, only the first variable is involved in the homogenization procedure, while the second plays the role of a parameter (see, for example, the definitions of $N$ and $M$, where this is literally the case). In particular, the hypothesis ensures that $N$ and $M$ belong to $H^{1}(\Xi)$ and that the pre-image of $L_{2}(\Xi)$ under $\mathcal{A}_{\mu}^{0}$ is $H^{2}(\Xi)$; as a consequence, the range of $\mathcal{K}_{\mu}^{\varepsilon}$ is contained in $H^{1}(\Xi)$.

Remark 8. While $\left(\mathcal{A}_{\mu}^{\varepsilon}\right)^{-1}$ and $\mathcal{D}_{2}\left(\mathcal{A}_{\mu}^{\varepsilon}\right)^{-1}$ have limits, the operator $\mathcal{D}_{1}\left(\mathcal{A}_{\mu}^{\varepsilon}\right)^{-1}$ can fail to converge, because, though the norm of $\varepsilon \mathcal{D}_{1} \mathcal{K}_{\mu}^{\varepsilon}$ is bounded uniformly in $\varepsilon$, it need not go to zero. However, if, for example, $\mathcal{D}_{1}^{*} A=0$ and $\mathcal{D}_{1}^{*} a_{2}=0$ (in the weak sense), then $\mathcal{K}_{\mu}^{\varepsilon}=0$, and $\mathcal{D}_{1}\left(\mathcal{A}_{\mu}^{\varepsilon}\right)^{-1}$ is therefore convergent as well. Notice that, in this case, the effective coefficients are obtained by simply taking the mean over $\Omega_{1}$.

Remark 9. We may replace $\mathcal{P}^{\varepsilon}$ with another smoothing. For instance, the Steklov averaging operator (see $[\mathrm{ZH}]$ ) or the scale-splitting operator (see [GR1]) can be used instead. This follows from the inequalities

$$
\begin{aligned}
\left\|\left(\mathcal{D}_{1} N\right) w\right\|_{2, \Xi}^{2} & \lesssim\left\|N \otimes \mathcal{D}_{1} w\right\|_{2, \Xi}^{2}+\|w\|_{2, \Xi}^{2} \\
\left\|\left(\mathcal{D}_{1} M\right) w\right\|_{2, \Xi}^{2} & \lesssim\left\|M \mathcal{D}_{1} w\right\|_{2, \Xi}^{2}+\|w\|_{1,2, \Xi}^{2}
\end{aligned}
$$

which hold for any $w \in C_{0}^{\infty}(\Xi)$ (the proof of the inequalities is parallel to that of Lemma 1), and properties of the smoothing operators (cf. similar techniques in [PSU, Lemma 3.5]). The reason why we chose $\mathcal{P}^{\varepsilon}$ is merely one of convenience: as we shall see below, $\mathcal{P}^{\varepsilon}$ takes a rather simple form after passing to the fundamental domain.

Remark 10. As already indicated, the operator $\mathcal{P}^{\varepsilon}$ guarantees that the range of $\mathcal{K}_{\mu}^{\varepsilon}$ is contained in $H^{1}(\Xi)$. This means that, in general, it is not possible to remove $\mathcal{P}^{\varepsilon}$. However, this can be done in certain cases. For example, if $N \in \mathbf{M}\left(L_{2}(\Omega)\right)^{1 \times d}$ and $M \in \mathbf{M}\left(H^{1}(\Omega), L_{2}(\Omega)\right)$, then the classical corrector

$$
\hat{\mathcal{K}}_{\mu}^{\varepsilon}=\left(N^{\varepsilon} \mathcal{D}+M^{\varepsilon}\right)\left(\mathcal{A}_{\mu}^{0}\right)^{-1}
$$

as well as the composition $\mathcal{D}_{1} \hat{\mathcal{K}}_{\mu}^{\varepsilon}$ are bounded on $L_{2}(\Xi)$ (by 36 ) and 37 ), and the estimates 34 and 35 remain true with $\hat{\mathcal{K}}_{\mu}^{\varepsilon}$ in place of $\mathcal{K}_{\mu}^{\varepsilon}$ and

$$
\hat{\mathcal{C}}_{\mu}^{\varepsilon}=\left(\hat{\mathcal{K}}_{\mu}^{\varepsilon}-\mathcal{L}_{\mu}\right)+\left(\left(\hat{\mathcal{K}}_{\mu}^{\varepsilon}\right)^{+}-\mathcal{L}_{\mu}^{+}\right)^{*}
$$

in place of $\mathcal{C}_{\mu}^{\varepsilon}$.

\section{Problem on the fundamental domain}

Our strategy is to reduce $\mathcal{A}_{\mu}^{\varepsilon}$ to an operator on the fundamental domain $\Omega$ and then formulate Theorems 13 in terms of this latter operator.

Let $\tau=(k, \varepsilon) \in \mathscr{T}=\Omega_{1}^{*} \times \mathscr{E}$. We introduce the notation $\mathcal{D}_{1}(\tau)=\mathcal{D}_{1}+\mathcal{K}$, $\mathcal{D}_{2}(\tau)=\varepsilon \mathcal{D}_{2}$ and $\mathcal{D}(\tau)=\mathcal{D}_{1}(\tau)+\mathcal{D}_{2}(\tau)$ and set

$$
\|u\|_{1,2, \Omega ; \tau}=\left(\|\mathcal{D}(\tau) u\|_{2, \Omega}^{2}+|\tau|^{2}\|u\|_{2, \Omega}^{2}\right)^{1 / 2}
$$


and

$$
\begin{aligned}
\|u\|_{1_{1}, 2, \Omega ; \tau} & =\left(\left\|\mathcal{D}_{1}(\tau) u\right\|_{2, \Omega}^{2}+|\tau|^{2}\|u\|_{2, \Omega}^{2}\right)^{1 / 2} \\
\|u\|_{1_{2}, 2, \Omega ; \tau} & =\left(\left\|\mathcal{D}_{2}(\tau) u\right\|_{2, \Omega}^{2}+|\tau|^{2}\|u\|_{2, \Omega}^{2}\right)^{1 / 2}
\end{aligned}
$$

for any $u$ for which the right-hand sides make sense.

Now let us define periodic Sobolev spaces over the interior of $\Omega$. Recall that we view $\Lambda$ as acting on $\Xi$ and $\Omega \subset \mathbb{R}^{d}$ is a fundamental domain for $\Lambda$. The space $\widetilde{W}_{p}^{m}(\Omega)$ consists of all functions that are in $W_{p}^{m}(\Omega)$ and that have periodic extensions in $W_{p}^{m}(K)$ for each compact set $K \subset \Xi$. Let $\widetilde{W}_{p, 0}^{m}(\Omega)$ be the subspace of functions in $\widetilde{W}_{p}^{m}(\Omega)$ with zero mean. As usual, $\widetilde{H}^{m}(\Omega)=\widetilde{W}_{2}^{m}(\Omega)$ and $\widetilde{H}_{0}^{m}(\Omega)=\widetilde{W}_{2,0}^{m}(\Omega)$.

We define the form $a(\tau)$ on $\widetilde{H}^{1}(\Omega)$ by

$$
\begin{aligned}
a(\tau)[u]= & (A \mathcal{D}(\tau) u, \mathcal{D}(\tau) u)_{2, \Omega}+\varepsilon\left(\mathcal{D}(\tau) u, a_{1} u\right)_{2, \Omega} \\
& +\varepsilon\left(a_{2} u, \mathcal{D}(\tau) u\right)_{2, \Omega}+\varepsilon^{2}(q u, u)_{2, \Omega} .
\end{aligned}
$$

Note that, when estimating $\left\|a_{n} u\right\|_{2, \Omega}, n \in[2]$, and $\left|(q u, u)_{2, \Omega}\right|$, we can replace $u$ by $u v$ with $v(x)=e^{i\left\langle x_{1}, k\right\rangle}$. Hence,

$$
\begin{aligned}
\varepsilon\left\|a_{n} u\right\|_{2, \Omega} & \leq\left\|a_{n}\right\|_{\mathbf{M}}\left(\varepsilon^{2}\left\|\mathcal{D}_{1}(\tau) u\right\|_{2, \Omega}^{2}+\left\|\mathcal{D}_{2}(\tau) u\right\|_{2, \Omega}^{2}+\varepsilon^{2}\|u\|_{2, \Omega}^{2}\right)^{1 / 2}, \\
\varepsilon^{2}\left|(q u, u)_{2, \Omega}\right| & \leq\|q\|_{\mathbf{M}}\left(\varepsilon^{2}\left\|\mathcal{D}_{1}(\tau) u\right\|_{2, \Omega}^{2}+\left\|\mathcal{D}_{2}(\tau) u\right\|_{2, \Omega}^{2}+\varepsilon^{2}\|u\|_{2, \Omega}^{2}\right) ;
\end{aligned}
$$

in particular, this means that

$$
\begin{aligned}
\varepsilon\left\|a_{n} u\right\|_{2, \Omega} & \leq\left\|a_{n}\right\|_{\mathbf{M}}\left(\|\mathcal{D}(\tau) u\|_{2, \Omega}^{2}+\varepsilon^{2}\|u\|_{2, \Omega}^{2}\right)^{1 / 2}, \\
\varepsilon^{2}\left|(q u, u)_{2, \Omega}\right| & \leq\|q\|_{\mathbf{M}}\left(\|\mathcal{D}(\tau) u\|_{2, \Omega}^{2}+\varepsilon^{2}\|u\|_{2, \Omega}^{2}\right)
\end{aligned}
$$

for all $u \in H^{1}(\Omega)$. Therefore, the same reasoning as for $a^{\varepsilon}$ gives

$$
|a(\tau)[u, v]| \leq C_{b}\|u\|_{1,2, \Omega ; \tau}\|v\|_{1,2, \Omega ; \tau}, \quad u, v \in \widetilde{H}^{1}(\Omega),
$$

and

$$
\operatorname{Re} \boldsymbol{a}(\tau)[u] \geq c_{*}\|\mathcal{D}(\tau) u\|_{2, \Omega}^{2}-\varepsilon^{2} c_{\natural}\|u\|_{2, \Omega}^{2}, \quad u \in \widetilde{H}^{1}(\Omega) .
$$

Define $\mathcal{A}_{\mu}(\tau)=\mathcal{A}(\tau)-\varepsilon^{2} \mu: \widetilde{H}^{1}(\Omega) \rightarrow \widetilde{H}^{1}(\Omega)^{*}$ to be the operator associated with the form $a_{\mu}(\tau)=a(\tau)-\varepsilon^{2} \mu$. It follows that $\mathcal{A}_{\mu}(\tau)$ is an isomorphism if $\mu \notin \delta_{1}$.

Lemma 3. For any $\mu \notin \mathcal{S}$ and $\tau \in \mathscr{T}$ we have

$$
\begin{aligned}
\left\|\left(\mathcal{A}_{\mu}(\tau)\right)^{-1}\right\|_{\mathbf{B}\left(L_{2}(\Omega)\right)} & \lesssim|\tau|^{-2}, \\
\left\|\mathcal{D}(\tau)\left(\mathcal{A}_{\mu}(\tau)\right)^{-1}\right\|_{\mathbf{B}\left(L_{2}(\Omega)\right)^{d}} & \lesssim|\tau|^{-1}, \\
\left\|\mathcal{D}(\tau)\left(\mathcal{A}_{\mu}(\tau)\right)^{-1} \mathcal{D}(\tau)\right\|_{\mathbf{B}\left(L_{2}(\Omega)\right)^{d \times d}} & \lesssim 1, \\
\left\|\mathcal{D}(\tau) \mathcal{D}_{2}(\tau)\left(\mathcal{A}_{\mu}(\tau)\right)^{-1}\right\|_{\mathbf{B}\left(L_{2}(\Omega)\right)^{d \times d}} & \lesssim 1,
\end{aligned}
$$

where the constants depend on $\mu$ and the multiplier norms of the coefficients.

Proof. We do the case $\mu \in \mathscr{R}$, where

$$
\mathscr{R}=\left\{z \in \mathbb{C}: \operatorname{Re} z<-c_{\natural}\right\} .
$$

The general case then follows by the resolvent identity.

Expanding $u \in \widetilde{H}^{1}(\Omega)$ in a Fourier series

$$
u(x)=\left|\Omega_{1}\right|^{-1 / 2} \sum_{\lambda^{*} \in \Lambda^{*}} \hat{u}_{\lambda^{*}}\left(x_{2}\right) e^{-i\left\langle x_{1}, \lambda^{*}\right\rangle},
$$


we find that, for all $k \in \Omega_{1}^{*}$,

$$
\left\|\mathcal{D}_{1}(\tau) u\right\|_{2, \Omega}^{2}=\sum_{\lambda^{*} \in \Lambda^{*}}\left|\lambda^{*}+k\right|^{2}\left\|\hat{u}_{\lambda^{*}}\right\|_{2, \Omega_{2}}^{2} \geq|k|^{2}\|u\|_{2, \Omega}^{2}
$$

which means that

$$
|\tau|^{2}\|u\|_{2, \Omega}^{2} \leq\|\mathcal{D}(\tau) u\|_{2, \Omega}^{2}+\varepsilon^{2}\|u\|_{2, \Omega}^{2} .
$$

Combining this with (44) gives the first estimate. The second is immediate from the first and (44), and the third follows at once from (44). It remains to prove the last.

We shall use the classical technique of difference quotients. To that end, we introduce a little notation. Let $e_{2, m}, m \in\left[d_{2}\right]$, be the unit vector along the $x_{2, m}$-axis. If $u$ is any function on $\Omega$, then we define the difference quotient $D_{2, m}^{h}$ in the variable $x_{2, m}$ of size $h \in \mathbb{R} \backslash\{0\}$ by setting $D_{2, m}^{h} u=-i h^{-1}\left(\mathcal{T}_{2, m}^{h} u-u\right)$ where $\left(\mathcal{T}_{2, m}^{h} u\right)(x)=u\left(x+h e_{2, m}\right)$. Observe that

$$
\left(D_{2, m}^{h}\right)^{*}=D_{2, m}^{-h}
$$

and

$$
D_{2, m}^{h}(u v)=\left(D_{2, m}^{h} u\right) \mathcal{T}_{2, m}^{h} v+u\left(D_{2, m}^{h} v\right)
$$

For $f \in L_{2}(\Omega)$ fixed, we set $w=\left(\mathcal{A}_{\mu}(\tau)\right)^{-1} f$. Then

$$
a_{\mu}(\tau)\left[D_{2, m}^{h} w\right]=\left(f, D_{2, m}^{-h} D_{2, m}^{h} w\right)_{2, \Omega}-\left(\left[D_{2, m}^{h}, \mathcal{A}_{\mu}(\tau)\right] w, D_{2, m}^{h} w\right)_{2, \Omega} .
$$

If we show that

$$
\left|\left(\left[D_{2, m}^{h}, \mathcal{A}_{\mu}(\tau)\right] u, v\right)_{2, \Omega}\right| \lesssim\|u\|_{1,2, \Omega ; \tau}\|v\|_{1,2, \Omega ; \tau}, \quad u, v \in \widetilde{H}^{1}(\Omega),
$$

where the constant is independent of $h$, then, by the estimates that we just proved, the right-hand side of (46) will not exceed

$$
\begin{aligned}
& \varepsilon^{-1}\left\|\mathcal{D}(\tau) D_{2, m}^{h} w\right\|_{2, \Omega}\|f\|_{2, \Omega}+|\tau|^{-1} C\left\|D_{2, m}^{h} w\right\|_{1,2, \Omega ; \tau}\|f\|_{2, \Omega} \\
& \quad \leq 2^{-1} c_{*}\left\|\mathcal{D}(\tau) D_{2, m}^{h} w\right\|_{2, \Omega}^{2}+\varepsilon^{2}\left|c_{\natural}+\operatorname{Re} \mu\right|\left\|D_{2, m}^{h} w\right\|_{2, \Omega}^{2}+\varepsilon^{-2} C\|f\|_{2, \Omega}^{2}
\end{aligned}
$$

with some constant $C$. We have used here the fact that, for any $h$,

$$
\left\|D_{2, m}^{-h} D_{2, m}^{h} w\right\|_{2, \Omega} \leq\left\|D_{2, m} D_{2, m}^{h} w\right\|_{2, \Omega} .
$$

On the other hand, since $-\left(c_{\natural}+\operatorname{Re} \mu\right)>0$, it follows from 444$)$ that

$$
\operatorname{Re} a_{\mu}(\tau)\left[D_{2, m}^{h} w\right] \geq c_{*}\left\|\mathcal{D}(\tau) D_{2, m}^{h} w\right\|_{2, \Omega}^{2}+\varepsilon^{2}\left|c_{\natural}+\operatorname{Re} \mu\right|\left\|D_{2, m}^{h} w\right\|_{2, \Omega}^{2} .
$$

As a result,

$$
\left\|\mathcal{D}(\tau) \varepsilon D_{2, m}^{h} w\right\|_{2, \Omega} \lesssim\|f\|_{2, \Omega}
$$

uniformly in $h$. Thus, there exists $\mathcal{D}(\tau) \mathcal{D}_{2, m}(\tau) w$, with

$$
\left\|\mathcal{D}(\tau) \mathcal{D}_{2, m}(\tau) w\right\|_{2, \Omega} \lesssim\|f\|_{2, \Omega}
$$

as desired.

We conclude by proving 477 . Since

$$
\begin{aligned}
\left(\left[D_{2, m}^{h}, \mathcal{A}_{\mu}(\tau)\right] \mathcal{T}_{2, m}^{-h} u, v\right)_{2, \Omega}= & \left(\left(D_{2, m}^{h} A\right) \mathcal{D}(\tau) u, \mathcal{D}(\tau) v\right)_{2, \Omega}-\varepsilon\left(\mathcal{D}(\tau) u,\left(D_{2, m}^{h} a_{1}\right) v\right)_{2, \Omega} \\
& +\varepsilon\left(\left(D_{2, m}^{h} a_{2}\right) u, \mathcal{D}(\tau) v\right)_{2, \Omega}+\varepsilon^{2}\left(\left(D_{2, m}^{h} q\right) u, v\right)_{2, \Omega},
\end{aligned}
$$


we see that it suffices to show that each coefficient of this form is still a multiplier with norm bounded by a constant independent of $h$, because then an argument similar to the one we used for proving (43) will lead to (47). Obviously,

$$
\left(D_{2, m}^{h} \gamma\right)(x)=\int_{(0,1)}\left(\mathcal{T}_{2, m}^{t h} D_{2, m} \gamma\right)(x) d t
$$

for every function $\gamma$ that has a derivative $D_{2, m} \gamma \in L_{2}(\Omega)$. A duality argument shows that if $D_{2, m} \gamma \in H^{1}(\Omega)^{*}$, then

$$
\left(\left(D_{2, m}^{h} \gamma\right) u, u\right)_{2, \Omega}=\int_{(0,1)}\left(\left(D_{2, m} \gamma\right) \mathcal{T}_{2, m}^{-t h} u, \mathcal{T}_{2, m}^{-t h} u\right)_{2, \Omega} d t
$$

any $u \in C^{1}(\bar{\Omega})$. Hence, $\left\|D_{2, m}^{h} A\right\|_{\mathbf{M}} \leq\left\|D_{2, m} A\right\|_{\mathbf{M}},\left\|D_{2, m}^{h} a_{n}\right\|_{\mathbf{M}} \leq\left\|D_{2, m} a_{n}\right\|_{\mathbf{M}}$, $n \in[2]$, and $\left\|D_{2, m}^{h} q\right\|_{\mathbf{M}} \leq\left\|D_{2, m} q\right\|_{\mathbf{M}}$. This completes the proof.

Let $\mathcal{A}_{\mu}(\tau)^{+}: \widetilde{H}^{1}(\Omega) \rightarrow \widetilde{H}^{1}(\Omega)^{*}$ be the the formal adjoint of $\mathcal{A}_{\mu}(\tau)$. From $(43)$ and 44 , we see that $\mathcal{A}_{\mu}(\tau)^{+}$is also an isomorphism whenever $\mu \notin \mathcal{S}_{1}$. Moreover, the conclusion of Lemma 3 holds for $\mathcal{A}_{\mu}(\tau)^{+}$. It is easy to note the relationship between $\mathcal{A}_{\mu}(\tau)$ and $\mathcal{A}_{\mu}(\tau)^{+}$. Indeed, a suitable restriction of $\mathcal{A}_{\mu}(\tau)^{+}$is the adjoint of the restriction of $\mathcal{A}_{\mu}(\tau)$, so that, if $\mu \notin \mathcal{S}_{1}$,

$$
\left(\left(\mathcal{A}_{\mu}(\tau)\right)^{-1} f, f\right)_{2, \Omega}=\left(f,\left(\mathcal{A}_{\mu}(\tau)^{+}\right)^{-1} f\right)_{2, \Omega}, \quad f \in L_{2}(\Omega)
$$

We shall think of $L_{2}(\Omega)$ as the tensor product $L_{2}\left(\Omega_{1}\right) \otimes L_{2}\left(\Omega_{2}\right)$. Recall that $\mathcal{G}$ is the Gelfand transform and $\mathcal{S}^{\varepsilon}$ is the scaling transformation. Clearly, $\mathcal{G S}^{\varepsilon} \otimes \mathcal{I}$ maps $H^{1}(\Xi)$ onto $L_{2}\left(\Omega_{1}^{*} ; \widetilde{H}^{1}(\Omega)\right)$ and, for any $u \in H^{1}(\Xi)$,

$$
a_{\mu}^{\varepsilon}[u]=\varepsilon^{-2} \int_{\Omega_{1}^{*}} a_{\mu}(\tau)[\tilde{u}(k, \cdot)] d k,
$$

where $\tilde{u}=\left(\mathcal{G S}^{\varepsilon} \otimes \mathcal{I}\right) u$; that is,

$$
\left(\mathcal{G S}^{\varepsilon} \otimes \mathcal{I}\right)\left(\mathcal{A}_{\mu}^{\varepsilon}\right)^{-1}\left(\mathcal{G S} \mathcal{S}^{\varepsilon} \otimes \mathcal{I}\right)^{-1}=\int_{\Omega_{1}^{*}}^{\oplus} \varepsilon^{2}\left(\mathcal{A}_{\mu}(\tau)\right)^{-1} d k
$$

Now, we would like to do the same for the operator $\mathcal{A}_{\mu}^{0}$. To this end, let $a^{0}(\tau)$ be the form on $\widetilde{H}^{1}(\Omega)$ defined by

$$
\begin{aligned}
a^{0}(\tau)[u]= & \left(A^{0} \mathcal{D}(\tau) u, \mathcal{D}(\tau) u\right)_{2, \Omega}+\varepsilon\left(\mathcal{D}(\tau) u, a_{1}^{0} u\right)_{2, \Omega} \\
& +\varepsilon\left(a_{2}^{0} u, \mathcal{D}(\tau) u\right)_{2, \Omega}+\varepsilon^{2}\left(q^{0} u, u\right)_{2, \Omega}
\end{aligned}
$$

where

$$
\left(q^{0} u, u\right)_{2, \Omega}=\int_{\Omega_{1}}\left(q^{0} u\left(x_{1}, \cdot\right), u\left(x_{1}, \cdot\right)\right)_{2, \Omega_{2}} d x_{1} .
$$

The same arguments used to obtain 25 and $(27)$ now show that

$$
\left|a^{0}(\tau)[u, v]\right| \leq C_{\mathrm{b}}^{0}\|u\|_{1,2, \Omega ; \tau}\|v\|_{1,2, \Omega ; \tau}, \quad u, v \in \widetilde{H}^{1}(\Omega),
$$

and

$$
\operatorname{Re} a^{0}(\tau)[u] \geq c_{*}\|\mathcal{D}(\tau) u\|_{2, \Omega}^{2}-\varepsilon^{2} c_{\natural}\|u\|_{2, \Omega}^{2}, \quad u \in \widetilde{H}^{1}(\Omega) .
$$

Let $\mathcal{A}_{\mu}^{0}(\tau)=\mathcal{A}^{0}(\tau)-\varepsilon^{2} \mu: \widetilde{H}^{1}(\Omega) \rightarrow \widetilde{H}^{1}(\Omega)^{*}$ be the operator corresponding to $a_{\mu}^{0}(\tau)=$ $a^{0}(\tau)-\varepsilon^{2} \mu$. Then $\mathcal{A}_{\mu}^{0}(\tau)$ is an isomorphism if $\mu \notin \mathcal{S}_{0}$. 
Lemma 4. For any $\mu \notin \mathcal{S}$ and $\tau \in \mathscr{T}$ we have

$$
\begin{aligned}
\left\|\left(\mathcal{A}_{\mu}^{0}(\tau)\right)^{-1}\right\|_{\mathbf{B}\left(L_{2}(\Omega)\right)} & \lesssim|\tau|^{-2}, \\
\left\|\mathcal{D}(\tau)\left(\mathcal{A}_{\mu}^{0}(\tau)\right)^{-1}\right\|_{\mathbf{B}\left(L_{2}(\Omega)\right)^{d}} & \lesssim|\tau|^{-1}, \\
\left\|\mathcal{D}(\tau)\left(\mathcal{A}_{\mu}^{0}(\tau)\right)^{-1} \mathcal{D}(\tau)\right\|_{\mathbf{B}\left(L_{2}(\Omega)\right)^{d \times d}} & \lesssim 1, \\
\left\|\mathcal{D}(\tau) \mathcal{D}(\tau)\left(\mathcal{A}_{\mu}^{0}(\tau)\right)^{-1}\right\|_{\mathbf{B}\left(L_{2}(\Omega)\right)^{d \times d}} & \lesssim 1,
\end{aligned}
$$

where the constants depend on $\mu$ and the multiplier norms of the coefficients.

Proof. The proof is similar to that of Lemma 3

Since, for every $u \in H^{1}(\Xi)$,

$$
a_{\mu}^{0}[u]=\varepsilon^{-2} \int_{\Omega_{1}^{*}} a_{\mu}^{0}(\tau)[\tilde{u}(k, \cdot)] d k,
$$

with $\tilde{u}=\left(\mathcal{G S}^{\varepsilon} \otimes \mathcal{I}\right) u$, it follows that

$$
\left(\mathcal{G S}^{\varepsilon} \otimes \mathcal{I}\right)\left(\mathcal{A}_{\mu}^{0}\right)^{-1}\left(\mathcal{G S}^{\varepsilon} \otimes \mathcal{I}\right)^{-1}=\int_{\Omega_{1}^{*}}^{\oplus} \varepsilon^{2}\left(\mathcal{A}_{\mu}^{0}(\tau)\right)^{-1} d k
$$

As a result, we may restate Theorem 1 in terms of the fibers $\mathcal{A}_{\mu}(\tau)$ and $\mathcal{A}_{\mu}^{0}(\tau)$.

Theorem 4. Let $\mu \notin \mathcal{S}$. Then for all $\tau \in \mathscr{T}$ it holds that

$$
\begin{aligned}
\left\|\left(\mathcal{A}_{\mu}(\tau)\right)^{-1}-\left(\mathcal{A}_{\mu}^{0}(\tau)\right)^{-1}\right\|_{\mathbf{B}\left(L_{2}(\Omega)\right)} & \lesssim|\tau|^{-1}, \\
\left\|\mathcal{D}_{2}(\tau)\left(\mathcal{A}_{\mu}(\tau)\right)^{-1}-\mathcal{D}_{2}(\tau)\left(\mathcal{A}_{\mu}^{0}(\tau)\right)^{-1}\right\|_{\mathbf{B}\left(L_{2}(\Omega)\right)^{d}} & \lesssim 1,
\end{aligned}
$$

where the constants depend only on $r_{\Lambda}, \mu$ and the multiplier norms of the coefficients.

Let $\mathcal{P}_{1}$ and $\mathcal{P}_{2}$ denote the orthogonal projections in $L_{2}(\Omega)$ onto $\mathbb{C} \otimes L_{2}\left(\Omega_{2}\right)$ and $L_{2}\left(\Omega_{1}\right) \otimes \mathbb{C}$, respectively. Notice that

$$
\left(\mathcal{G S} \mathcal{S}^{\varepsilon} \otimes \mathcal{I}\right) \mathcal{P}^{\varepsilon}\left(\mathcal{G S}^{\varepsilon} \otimes \mathcal{I}\right)^{-1}=\int_{\Omega_{1}^{*}}^{\oplus} \mathcal{P}_{1} d k
$$

Define $\mathcal{K}_{\mu}(\tau): L_{2}(\Omega) \rightarrow \widetilde{H}^{1}(\Omega)$ by

$$
\mathcal{K}_{\mu}(\tau)=(N \mathcal{D}(\tau)+\varepsilon M)\left(\mathcal{A}_{\mu}^{0}(\tau)\right)^{-1} \mathcal{P}_{1} .
$$

Lemma 5. For any $\mu \notin \mathcal{S}$ and $\tau \in \mathscr{T}$ we have

$$
\begin{aligned}
\left\|\mathcal{D}_{1} \mathcal{K}_{\mu}(\tau)\right\|_{\mathbf{B}\left(L_{2}(\Omega)\right)^{d}} & \lesssim|\tau|^{-1}, \\
\left\|\mathcal{D}_{1} \mathcal{D}_{2}(\tau) \mathcal{K}_{\mu}(\tau)\right\|_{\mathbf{B}\left(L_{2}(\Omega)\right)^{d \times d}} & \lesssim 1,
\end{aligned}
$$

where the constants depend on $\mu$ and the multiplier norms of the coefficients.

Proof. Let $f \in L_{2}(\Omega)$, and let $u=\left(\mathcal{A}_{\mu}^{0}(\tau)\right)^{-1} \mathcal{P}_{1} f$ and $U=\mathcal{K}_{\mu}(\tau) f$. Then, by Lemma 4 , it follows that

$$
\left\|\mathcal{D}_{1} U\right\|_{2, \Omega} \leq\left|\Omega_{1}\right|^{-1 / 2}\left(\left\|\mathcal{D}_{1} N\right\|_{\mathbf{M}}+\left\|\mathcal{D}_{1} M\right\|_{\mathbf{M}}\right)\|u\|_{1_{2}, 2, \Omega ; \tau} \lesssim|\tau|^{-1}\|f\|_{2, \Omega}
$$

and

$$
\begin{aligned}
\left\|\mathcal{D}_{1} \mathcal{D}_{2}(\tau) U\right\|_{2, \Omega} \leq & \left|\Omega_{1}\right|^{-1 / 2}\left(\left\|\mathcal{D}_{1} \mathcal{D}_{2} N\right\|_{\mathbf{M}}+\left\|\mathcal{D}_{1} \mathcal{D}_{2} M\right\|_{\mathbf{M}}\right)|\tau|\|u\|_{1_{2}, 2, \Omega ; \tau} \\
& +\left|\Omega_{1}\right|^{-1 / 2}\left(\left\|\mathcal{D}_{1} N\right\|_{\mathbf{M}}+\left\|\mathcal{D}_{1} M\right\|_{\mathbf{M}}\right)\left\|\mathcal{D}_{2}(\tau) u\right\|_{1_{2}, 2, \Omega ; \tau} \lesssim\|f\|_{2, \Omega},
\end{aligned}
$$

as required. 
We remark that, since $\mathcal{P}_{1} \mathcal{K}_{\mu}(\tau)=0$ (by the definitions of $N$ and $M$ ), we may use Poincaré's inequality to see that $\mathcal{K}_{\mu}(\tau)$ and $\mathcal{D}_{2}(\tau) \mathcal{K}_{\mu}(\tau)$ satisfy estimates similar to those for $\mathcal{D}_{1} \mathcal{K}_{\mu}(\tau)$ and $\mathcal{D}_{1} \mathcal{D}_{2}(\tau) \mathcal{K}_{\mu}(\tau)$, respectively. This means that, unlike the case of $\mathcal{A}_{\mu}(\tau)$ and $\mathcal{A}_{\mu}^{0}(\tau)$, where both $\mathcal{D}_{1}(\tau)$ and $\mathcal{D}_{2}(\tau)$ make the norms of the corresponding compositions smaller, roughly speaking, by multiplying each of these norms by $|\tau|$, the differentiation $\mathcal{D}_{1}$ will not change the order of the norm of $\mathcal{K}_{\mu}(\tau)$. The reason, of course, is that the corrector $\mathcal{K}_{\mu}^{\varepsilon}$ involves functions that rapidly oscillate in the first variable.

In the same fashion as above, we may prove that

$$
\left(\mathcal{G S}^{\varepsilon} \otimes \mathcal{I}\right) \mathcal{K}_{\mu}^{\varepsilon}\left(\mathcal{G S}^{\varepsilon} \otimes \mathcal{I}\right)^{-1}=\int_{\Omega_{1}^{*}}^{\oplus} \varepsilon \mathcal{K}_{\mu}(\tau) d k
$$

Theorem 2 now takes the following form:

Theorem 5. Let $\mu \notin \mathcal{S}$. Then for all $\tau \in \mathscr{T}$ it holds that

$$
\left\|\mathcal{D}_{1}(\tau)\left(\mathcal{A}_{\mu}(\tau)\right)^{-1}-\mathcal{D}_{1}(\tau)\left(\mathcal{A}_{\mu}^{0}(\tau)\right)^{-1}-\mathcal{D}_{1}(\tau) \mathcal{K}_{\mu}(\tau)\right\|_{\mathbf{B}\left(L_{2}(\Omega)\right)^{d}} \lesssim 1,
$$

where the constant depends only on $r_{\Lambda}, \mu$ and the multiplier norms of the coeffcients.

Let $\mathcal{S}(\tau): \widetilde{H}^{1}(\Omega) \rightarrow \widetilde{H}^{1}(\Omega)^{*}$ and $\mathcal{T}(\tau): \widetilde{H}^{1}(\Omega) \rightarrow \widetilde{H}^{1}(\Omega)^{*}$ be given by

$$
\begin{aligned}
& \mathcal{S}(\tau)=\left(\left(\mathcal{K}+\mathcal{D}_{2}(\tau)\right)^{*} A+\varepsilon a_{1}^{*}\right)\left(\mathcal{K}+\mathcal{D}_{2}(\tau)\right)+\varepsilon\left(\mathcal{K}+\mathcal{D}_{2}(\tau)\right)^{*} a_{2}+\varepsilon^{2} q, \\
& \mathcal{T}(\tau)=\left(\left(\mathcal{K}+\mathcal{D}_{2}(\tau)\right)^{*} A+\varepsilon a_{1}^{*}\right) \mathcal{D}_{1} .
\end{aligned}
$$

Clearly, $\mathcal{S}(\tau)$ and $\mathcal{T}(\tau)$ are bounded operators, satisfying estimates like that for $\mathcal{A}_{\mu}(\tau)$. It is in fact possible to improve these estimates by using $(39)$ and 400 instead of (41) and 42):

(58) $\left|(\mathcal{S}(\tau) u, v)_{2, \Omega}\right| \lesssim\left(\varepsilon\left\|\mathcal{D}_{1}(\tau) u\right\|_{2, \Omega}+\|u\|_{1_{2}, 2, \Omega ; \tau}\right)\left(\varepsilon\left\|\mathcal{D}_{1}(\tau) v\right\|_{2, \Omega}+\|v\|_{1_{2}, 2, \Omega ; \tau}\right)$,

$$
\left|(\mathcal{T}(\tau) u, v)_{2, \Omega}\right| \lesssim\left\|\mathcal{D}_{1} u\right\|_{2, \Omega}\left(\varepsilon\left\|\mathcal{D}_{1}(\tau) v\right\|_{2, \Omega}+\|v\|_{1_{2}, 2, \Omega ; \tau}\right)
$$

if $u, v \in \widetilde{H}^{1}(\Omega)$. The operators $\mathcal{S}(\tau)^{+}$and $\mathcal{T}(\tau)^{+}$are defined likewise. Of course, estimates similar to 58 and 59 hold for $\mathcal{S}(\tau)^{+}$and $\mathcal{T}(\tau)^{+}$as well. Notice that

$$
\mathcal{A}_{\mu}(\tau)=\mathcal{D}_{1}^{*} A \mathcal{D}_{1}+\mathcal{S}(\tau)+\mathcal{T}(\tau)+\left(\mathcal{T}(\tau)^{+}\right)^{*}-\varepsilon^{2} \mu .
$$

We break Theorem 3 into two parts. The first is formulated as follows:

Lemma 6. For any $\mu \notin \mathcal{S}$ and $\varepsilon \in \mathscr{E}$ we have

$$
\left\|\mathcal{L}_{\mu}\left(\mathcal{I}-\mathcal{P}^{\varepsilon}\right)\right\|_{\mathbf{B}\left(L_{2}(\Xi)\right)} \lesssim \varepsilon
$$

where the constant depends on $r_{\Lambda}$, $\mu$ and the multiplier norms of the coefficients.

Proof. We estimate the operator norm of the symbol $\mathcal{L}_{\mu}(\cdot)$. Fix $k \in \mathbb{R}^{d_{1}} \backslash\{0\}$. Let $f \in L_{2}\left(\Omega_{2}\right)$, and let $u=\left(\mathcal{A}_{\mu}^{0}(\tau)\right)^{-1} f$ and $U=\mathcal{K}_{\mu}(\tau) f, U^{+}=\mathcal{K}_{\mu}(\tau)^{+} f$ where $\tau=(k, 1)$. Then

$$
\left(\mathcal{L}_{\mu}(k) f, f\right)_{2, \Omega_{2}}=\left|\Omega_{1}\right|^{-1}\left(\mathcal{S}(\tau) u+\mathcal{T}(\tau) U, U^{+}\right)_{2, \Omega} .
$$

It should be noted that while Lemmas 4 and 5 are only asserted to be valid for $\mathcal{A}_{\mu}^{0}(\tau)$ and $\mathcal{K}_{\mu}(\tau)$ with $\tau \in \Omega_{1}^{*} \times \mathscr{E}$, they may be extended to $\mathcal{A}_{\mu}^{0}(\tau) \mathcal{P}_{1}$ and $\mathcal{K}_{\mu}(\tau)$ with $\tau \in$ $\mathbb{R}^{d_{1}} \times \mathscr{E}$. Indeed, the condition $k \in \Omega_{1}^{*}$ is used only to ensure the inequality 45). But when $u$ does not depend on $x_{1}$, we have equality for each $k \in \mathbb{R}^{d_{1}}$. Thus, the 
estimates (58) and (59) together with these extended versions of Lemmas 4 and 5 . as well as Poincaré's inequality, give

$$
\left|\left(\mathcal{L}_{\mu}(k) f, f\right)_{2, \Omega_{2}}\right| \lesssim\left(\|u\|_{1_{2}, 2, \Omega ; \tau}+\left\|\mathcal{D}_{1} U\right\|_{2, \Omega}\right)\left\|\mathcal{D}_{1} U^{+}\right\|_{1_{2}, 2, \Omega ; \tau} \lesssim|k|^{-1}\|f\|_{2, \Omega_{2}}^{2}
$$

Now if $g \in C_{0}^{\infty}(\Xi)$ and $\hat{g}=(\mathcal{F} \otimes \mathcal{I}) g$, then

$$
\begin{aligned}
\left|\left(\mathcal{L}_{\mu}\left(\mathcal{I}-\mathcal{P}^{\varepsilon}\right) g, g\right)_{2, \Xi}\right| & \leq \int_{\mathbb{R}^{d_{1}} \backslash \varepsilon^{-1} \Omega_{1}^{*}}\left|\left(\mathcal{L}_{\mu}(k) \hat{g}(k, \cdot), \hat{g}(k, \cdot)\right)_{2, \Omega_{2}}\right| d k \\
& \lesssim \int_{\mathbb{R}^{d_{1}} \backslash \varepsilon^{-1} \Omega_{1}^{*}}|k|^{-1}\|\hat{g}(k, \cdot)\|_{2, \Omega}^{2} d k \leq \varepsilon r_{\Lambda}^{-1}\|g\|_{2, \Xi}^{2} .
\end{aligned}
$$

This is the result that we wished to prove.

The lemma takes care of $\mathcal{L}_{\mu}\left(\mathcal{I}-\mathcal{P}^{\varepsilon}\right)$ and $\mathcal{L}_{\mu}^{+}\left(\mathcal{I}-\mathcal{P}^{\varepsilon}\right)$ in the estimate 35 , so we may concentrate our attention on $\mathcal{L}_{\mu} \mathcal{P}^{\varepsilon}$ and $\mathcal{L}_{\mu}^{+} \mathcal{P}^{\varepsilon}$. Let $\mathcal{A}_{\mu}^{0}(\tau)^{+}$and $\mathcal{K}_{\mu}(\tau)^{+}$play the roles of $\mathcal{A}_{\mu}^{0}(\tau)$ and $\mathcal{K}_{\mu}(\tau)$ for $\mathcal{A}_{\mu}(\tau)^{+}$. Then, since

$$
\left(\left(\mathcal{G S} \mathcal{S}^{\varepsilon} \otimes \mathcal{I}\right) \mathcal{P}^{\varepsilon} u\right)(k, x)=\left|\Omega_{1}\right|^{-1 / 2}\left(\left(\mathcal{S}^{1 / \varepsilon} \mathcal{F} \otimes \mathcal{I}\right) u\right)\left(k, x_{2}\right)
$$

for every $u \in L_{2}(\Xi)$, we find that

$$
\left(\mathcal{G S}^{\varepsilon} \otimes \mathcal{I}\right) \mathcal{L}_{\mu} \mathcal{P}^{\varepsilon}\left(\mathcal{G} \mathcal{S}^{\varepsilon} \otimes \mathcal{I}\right)^{-1}=\int_{\Omega_{1}^{*}}^{\oplus} \varepsilon \mathcal{L}_{\mu}(\tau) \mathcal{P}_{1} d k
$$

where $\mathcal{L}_{\mu}(\tau): L_{2}(\Omega) \rightarrow L_{2}(\Omega)$ is given by

$$
\mathcal{L}_{\mu}(\tau)=\left(\mathcal{K}_{\mu}(\tau)^{+}\right)^{*}\left(\mathcal{S}(\tau)\left(\mathcal{A}_{\mu}^{0}(\tau)\right)^{-1}+\mathcal{T}(\tau) \mathcal{K}_{\mu}(\tau)\right) .
$$

Define $\mathcal{L}_{\mu}(\tau)^{+}$similarly. Obviously, in order to prove Theorem 3 we need to establish the following result:

Theorem 6. Let $\mu \notin \mathcal{S}$. Then for all $\tau \in \mathscr{T}$ it holds that

$$
\left\|\left(\mathcal{A}_{\mu}(\tau)\right)^{-1}-\left(\mathcal{A}_{\mu}^{0}(\tau)\right)^{-1}-\left(\mathcal{K}_{\mu}(\tau)-\mathcal{L}_{\mu}(\tau)\right) \mathcal{P}_{1}-\mathcal{P}_{1}\left(\mathcal{K}_{\mu}(\tau)^{+}-\mathcal{L}_{\mu}(\tau)^{+}\right)^{*}\right\|_{\mathbf{B}\left(L_{2}(\Omega)\right)} \lesssim 1,
$$

where the constant depends only on $r_{\Lambda}, \mu$ and the multiplier norms of the coeffcients.

We now turn to the proofs. Our first goal is to verify the identity

$$
\begin{aligned}
\left(\mathcal{A}_{\mu}(\tau)\right)^{-1} \mathcal{P}_{1}-\left(\mathcal{A}_{\mu}^{0}(\tau)\right)^{-1} \mathcal{P}_{1}-\mathcal{K}_{\mu}(\tau) & \\
= & -\left(\mathcal{A}_{\mu}(\tau)\right)^{-1} \mathcal{P}_{1}^{\perp}\left(\mathcal{S}(\tau)\left(\mathcal{A}_{\mu}^{0}(\tau)\right)^{-1} \mathcal{P}_{1}+\mathcal{T}(\tau) \mathcal{K}_{\mu}(\tau)\right) \\
& -\left(\mathcal{A}_{\mu}(\tau)\right)^{-1}\left(\mathcal{S}(\tau)^{+}+\mathcal{T}(\tau)^{+}-\varepsilon^{2} \bar{\mu}\right)^{*} \mathcal{K}_{\mu}(\tau)
\end{aligned}
$$

Denote the operator on the left by $\mathcal{U}_{\mu}(\tau)$. If $f, g \in L_{2}(\Omega)$, then we set $u=$ $\left(\mathcal{A}_{\mu}^{0}(\tau)\right)^{-1} f, U=\mathcal{K}_{\mu}(\tau) f$ and $v^{+}=\left(\mathcal{A}_{\mu}(\tau)^{+}\right)^{-1} g$. By 48$)$, we have

$$
\left(\mathcal{U}_{\mu}(\tau) f, g\right)_{2, \Omega}=a_{\mu}^{0}(\tau)\left[\mathcal{P}_{1} u, v^{+}\right]-a_{\mu}(\tau)\left[\mathcal{P}_{1} u+U, v^{+}\right]
$$

(and here we are using the fact that $\mathcal{P}_{1}$ commutes with $\mathcal{D}(\tau)$ on periodic functions). Looking at the definitions of the effective coefficients, we see that

$$
\mathcal{A}_{\mu}^{0}(\tau) \mathcal{P}_{1}=\mathcal{P}_{1}\left(\mathcal{A}_{\mu}(\tau)+\mathcal{T}(\tau)(N \mathcal{D}(\tau)+\varepsilon M)\right) \mathcal{P}_{1},
$$

from which we obtain

$$
a_{\mu}^{0}(\tau)\left[\mathcal{P}_{1} u, v^{+}\right]-a_{\mu}(\tau)\left[\mathcal{P}_{1} u, v^{+}\right]=\left(\mathcal{T}(\tau) U, \mathcal{P}_{1} v^{+}\right)_{2, \Omega}-\left(\mathcal{A}_{\mu}(\tau) \mathcal{P}_{1} u, \mathcal{P}_{1}^{\perp} v^{+}\right)_{2, \Omega}
$$


On the other hand, it follows from 60 that

$$
a_{\mu}(\tau)\left[U, v^{+}\right]=\left(\mathcal{T}(\tau) U, \mathcal{P}_{1} v^{+}\right)_{2, \Omega}+\left(\mathcal{S}(\tau) U, \mathcal{P}_{1} v^{+}\right)_{2, \Omega}+\left(\mathcal{A}_{\mu}(\tau) U, \mathcal{P}_{1}^{\perp} v^{+}\right)_{2, \Omega}
$$

The first term on the right-hand side of this last equality cancels with the first term on the right-hand side of 65, so

$$
\left(\mathcal{U}_{\mu}(\tau) f, g\right)_{2, \Omega}=-\left(\mathcal{A}_{\mu}(\tau)\left(\mathcal{P}_{1} u+U\right), \mathcal{P}_{1}^{\perp} v^{+}\right)_{2, \Omega}-\left(\mathcal{S}(\tau) U, \mathcal{P}_{1} v^{+}\right)_{2, \Omega}
$$

Notice that, by the definitions of $N$ and $M$,

$$
\mathcal{D}_{1}^{*} A \mathcal{D}_{1} U+\left(\mathcal{T}(\tau)^{+}\right)^{*} \mathcal{P}_{1} u=0 .
$$

Since $\mathcal{T}(\tau) \mathcal{P}_{1}=0$ and $\mathcal{P}_{1} \mathcal{K}_{\mu}(\tau)=0$, this and the identity 60 imply that

$\left(\mathcal{U}_{\mu}(\tau) f, g\right)_{2, \Omega}=-\left(\mathcal{S}(\tau) \mathcal{P}_{1} u+\mathcal{T}(\tau) U, \mathcal{P}_{1}^{\perp} v^{+}\right)_{2, \Omega}-\left(\mathcal{S}(\tau) U+\left(\mathcal{T}(\tau)^{+}\right)^{*} U-\varepsilon^{2} \mu U, v^{+}\right)_{2, \Omega}$.

Then, using (48) and the fact that $\mathcal{S}(\tau)^{+}$is the formal adjoint of $\mathcal{S}(\tau)$, we get 63.

$$
\begin{aligned}
\left(\mathcal{A}_{\mu}(\tau)\right)^{-1} \mathcal{P}_{1}-\left(\mathcal{A}_{\mu}^{0}(\tau)\right)^{-1} \mathcal{P}_{1}-\mathcal{K}_{\mu}(\tau)+\mathcal{L}_{\mu}(\tau) \mathcal{P}_{1}+\mathcal{P}_{1}\left(\mathcal{L}_{\mu}(\tau)^{+}\right)^{*} \\
=-\left(\left(\mathcal{A}_{\mu}(\tau)^{+}\right)^{-1}-\mathcal{K}_{\mu}(\tau)^{+}\right)^{*} \mathcal{P}_{1}^{\perp}\left(\mathcal{S}(\tau)\left(\mathcal{A}_{\mu}^{0}(\tau)\right)^{-1} \mathcal{P}_{1}+\mathcal{T}(\tau) \mathcal{K}_{\mu}(\tau)\right) \\
\quad-\left(\left(\mathcal{A}_{\mu}(\tau)^{+}\right)^{-1}-\left(\mathcal{A}_{\mu}^{0}(\tau)^{+}\right)^{-1} \mathcal{P}_{1}-\mathcal{K}_{\mu}(\tau)^{+}\right)^{*}\left(\mathcal{S}(\tau)^{+}+\mathcal{T}(\tau)^{+}\right)^{*} \mathcal{K}_{\mu}(\tau) \\
\quad-\left(\mathcal{S}(\tau)^{+} \mathcal{K}_{\mu}(\tau)^{+}-\varepsilon^{2} \bar{\mu}\left(\mathcal{A}_{\mu}(\tau)^{+}\right)^{-1}\right)^{*} \mathcal{K}_{\mu}(\tau)
\end{aligned}
$$

To prove this, we just note that $\mathcal{T}(\tau)^{+} \mathcal{P}_{1}=0$ and $\mathcal{P}_{1} \mathcal{K}_{\mu}(\tau)=0$ and then apply 63. We denote the operator on the left by $\mathcal{V}_{\mu}(\tau)$.

With these results in hand, it is easy to complete the proofs of the theorems.

Proof of Theorem 4. We write

$$
\left(\mathcal{A}_{\mu}(\tau)\right)^{-1}-\left(\mathcal{A}_{\mu}^{0}(\tau)\right)^{-1}=\mathcal{U}_{\mu}(\tau)+\left(\mathcal{A}_{\mu}(\tau)\right)^{-1} \mathcal{P}_{1}^{\perp}-\left(\mathcal{A}_{\mu}^{0}(\tau)\right)^{-1} \mathcal{P}_{1}^{\perp}+\mathcal{K}_{\mu}(\tau) .
$$

By Poincaré's inequality (5) and Lemmas 3 and 4 (for $\mathcal{A}_{\mu}(\tau)^{+}$and $\mathcal{A}_{\mu}^{0}(\tau)^{+}$, respectively), the norms of $|\tau|\left(\mathcal{A}_{\mu}(\tau)\right)^{-1} \mathcal{P}_{1}^{\perp}, \mathcal{D}_{2}(\tau)\left(\mathcal{A}_{\mu}(\tau)\right)^{-1} \mathcal{P}_{1}^{\perp}$ as well as $|\tau|\left(\mathcal{A}_{\mu}^{0}(\tau)\right)^{-1} \mathcal{P}_{1}^{\perp}$, $\mathcal{D}_{2}(\tau)\left(\mathcal{A}_{\mu}^{0}(\tau)\right)^{-1} \mathcal{P}_{1}^{\perp}$ are uniformly bounded. In Lemma 5 , we proved that so are the norms of $|\tau| \mathcal{K}_{\mu}(\tau)$ and $\mathcal{D}_{2}(\tau) \mathcal{K}_{\mu}(\tau)$. Thus, it is enough to show that

$$
\begin{aligned}
\left\|\mathcal{U}_{\mu}(\tau)\right\|_{\mathbf{B}\left(L_{2}(\Omega)\right)} & \lesssim|\tau|^{-1}, \\
\left\|\mathcal{D}_{2}(\tau) \mathcal{U}_{\mu}(\tau)\right\|_{\mathbf{B}\left(L_{2}(\Omega)\right)} & \lesssim 1 .
\end{aligned}
$$

Let notation be as above. We use $(58)$ and $(59)$ together with the Poincaré inequality (5) to estimate each term in 63). The result is that

$$
\begin{aligned}
\left|\left(\mathcal{U}_{\mu}(\tau) f, g\right)_{2, \Omega}\right| \lesssim|\tau|^{-1}\left(|\tau|\|u\|_{1_{2}, 2, \Omega ; \tau}+\left\|\mathcal{D}_{1} U\right\|_{1_{2}, 2, \Omega ; \tau}\right) \\
\\
\times\left(\left\|\mathcal{D}_{1}(\tau) v^{+}\right\|_{1_{2}, 2, \Omega ; \tau}+|\tau|\left\|v^{+}\right\|_{1_{2}, 2, \Omega ; \tau}\right) .
\end{aligned}
$$

Combining this with Lemmas 3 (for $\left.\mathcal{A}_{\mu}(\tau)^{+}\right), 4$ and 5 gives 68 .

The inequality 69 is proved in a like manner. We set $w^{+}=\left(\mathcal{A}_{\mu}(\tau)^{+}\right)^{-1} \mathcal{D}_{2}(\tau)^{*} g$ with $g \in L_{2}(\Omega)^{d}$ such that $\mathcal{D}_{2}(\tau)^{*} g \in L_{2}(\Omega)$ and then estimate the form

$$
\begin{aligned}
\left(\mathcal{U}_{\mu}(\tau) f, \mathcal{D}_{2}(\tau)^{*} g\right)_{2, \Omega}= & -\left(\mathcal{S}(\tau) \mathcal{P}_{1} u+\mathcal{T}(\tau) U, \mathcal{P}_{1}^{\perp} w^{+}\right)_{2, \Omega} \\
& -\left(U,\left(\mathcal{S}(\tau)^{+}+\mathcal{T}(\tau)^{+}-\varepsilon^{2} \bar{\mu}\right) w^{+}\right)_{2, \Omega} .
\end{aligned}
$$


However, a modification is required to eliminate the mixed second derivatives of $w^{+}$ which arise when we estimate the right-hand side (cf. (70), where a similar term, namely $\mathcal{D}_{2}(\tau) \mathcal{D}_{1}(\tau) v^{+}$, causes no difficulty).

We do so as follows. Let $\varphi \in L_{2}\left(\Omega_{2} ; \widetilde{H}^{1}\left(\Omega_{1}\right)\right)$, and let $\psi$ be the solution of

$$
\mathcal{D}_{2}^{*}(\tau) \mathcal{D}_{2}(\tau) \psi+|\tau|^{2} \psi=\varphi
$$

in $L_{2}\left(\Omega_{1} ; H^{1}\left(\Omega_{2}\right)\right)$. Obviously, $\psi$ has first derivatives and mixed second derivatives, as well as pure second derivatives in $x_{2}$. Fix $l \in \mathbb{R}^{d_{1}} \oplus\{0\}$ with $|\mathcal{L}|=|\tau|$ and define the operator $\mathcal{E}(\tau): L_{2}\left(\Omega_{2} ; \widetilde{H}^{1}\left(\Omega_{1}\right)\right) \rightarrow \widetilde{H}^{1}(\Omega)^{d}$ that assigns to each $\varphi \in$ $L_{2}\left(\Omega_{2} ; \widetilde{H}^{1}\left(\Omega_{1}\right)\right)$ the function $\left(\complement+\mathcal{D}_{2}(\tau)\right) \psi$. It follows that $\left(\mathcal{L}+\mathcal{D}_{2}(\tau)\right)^{*} \mathcal{E}(\tau)$ is the identity mapping. A straightforward calculation (using the fact that $\|\mathcal{E}(\tau) \varphi\|_{2, \Omega}=$ $\left.\|\psi\|_{1_{2}, 2, \Omega ; \tau}\right)$ shows that $\mathcal{E}(\tau)$ is bounded and

$$
|\tau|\left\|\mathcal{D}_{1}(\tau) \mathcal{E}(\tau) \varphi\right\|_{2, \Omega}+\|\mathcal{E}(\tau) \varphi\|_{1_{2}, 2, \Omega ; \tau} \leq\left\|\mathcal{D}_{1}(\tau) \varphi\right\|_{2, \Omega}+3\|\varphi\|_{2, \Omega} .
$$

Now, we may rewrite the first expression on the right-hand side of (71) as

$$
\begin{aligned}
\left(\mathcal{S}(\tau) \mathcal{P}_{1} u+\mathcal{T}(\tau) U, \mathcal{P}_{1}^{\perp} w^{+}\right)_{2, \Omega} \\
=\varepsilon\left(\left[\mathcal{D}_{2}, \mathcal{S}(\tau)\right] \mathcal{P}_{1} u+\left[\mathcal{D}_{2}, \mathcal{T}(\tau)\right] U, \mathcal{E}(\tau) \mathcal{P}_{1}^{\perp} w^{+}\right)_{2, \Omega} \\
\quad+\left(\mathcal{S}(\tau)\left(\mathcal{L}+\mathcal{D}_{2}(\tau)\right) \mathcal{P}_{1} u+\mathcal{T}(\tau)\left(\mathcal{L}+\mathcal{D}_{2}(\tau)\right) U, \mathcal{E}(\tau) \mathcal{P}_{1}^{\perp} w^{+}\right)_{2, \Omega} .
\end{aligned}
$$

Applying (58) and (59) and similar results for the commutators of $\mathcal{D}_{2}$ with $\mathcal{S}(\tau)$ and $\mathcal{T}(\tau)$ (notice that these commutators have the same forms as $\mathcal{S}(\tau)$ and $\mathcal{T}(\tau)$ ), we conclude that

$$
\begin{aligned}
\left|\left(\mathcal{S}(\tau) \mathcal{P}_{1} u+\mathcal{T}(\tau) U, \mathcal{P}_{1}^{\perp} w^{+}\right)_{2, \Omega}\right| \\
\lesssim\left(\left\|\mathcal{D}_{2}(\tau) u\right\|_{1_{2}, 2, \Omega ; \tau}+|\tau|\|u\|_{1_{2}, 2, \Omega ; \tau}+\left\|\mathcal{D}_{1} U\right\|_{1_{2}, 2, \Omega ; \tau}\right) \\
\quad \times\left(|\tau|\left\|\mathcal{D}_{1}(\tau) \mathcal{E}(\tau) \mathcal{P}_{1}^{\perp} w^{+}\right\|_{2, \Omega}+\left\|\mathcal{E}(\tau) \mathcal{P}_{1}^{\perp} w^{+}\right\|_{1_{2}, 2, \Omega ; \tau}\right)
\end{aligned}
$$

Therefore, by the estimate 72 together with the Poincaré inequality,

$$
\begin{aligned}
& \left|\left(\mathcal{S}(\tau) \mathcal{P}_{1} u+\mathcal{T}(\tau) U, \mathcal{P}_{1}^{\perp} w^{+}\right)_{2, \Omega}\right| \\
& \quad \lesssim\left(\left\|\mathcal{D}_{2}(\tau) u\right\|_{1_{2}, 2, \Omega ; \tau}+|\tau|\|u\|_{1_{2}, 2, \Omega ; \tau}+\left\|\mathcal{D}_{1} U\right\|_{1_{2}, 2, \Omega ; \tau}\right)\left\|\mathcal{D}_{1}(\tau) w^{+}\right\|_{2, \Omega} .
\end{aligned}
$$

The second expression is handled in the same way as before. The upshot is that

$$
\begin{aligned}
& \quad\left|\left(\mathcal{U}_{\mu}(\tau) f, \mathcal{D}_{2}(\tau)^{*} g\right)_{2, \Omega}\right| \\
& \quad \lesssim\left(\left\|\mathcal{D}_{2}(\tau) u\right\|_{1_{2}, 2, \Omega ; \tau}+|\tau|\|u\|_{1_{2}, 2, \Omega ; \tau}+\left\|\mathcal{D}_{1} U\right\|_{1_{2}, 2, \Omega ; \tau}\right)\left\|w^{+}\right\|_{1,2, \Omega ; \tau},
\end{aligned}
$$

whence 69 follows by Lemmas $3\left(\right.$ for $\left.\mathcal{A}_{\mu}(\tau)^{+}\right), 4$ and 5 .

Proof of Theorem 5. The proof follows the same pattern as the previous one. Again, the assertion is reduced, by Poincaré's inequality and Lemmas 3 and 4 . to the estimation of $\mathcal{D}_{1}(\tau) \mathcal{U}_{\mu}(\tau)$. Then the arguments that we used to obtain 74 go through without change to yield the desired conclusion.

Proof of Theorem 6. We write

$$
\begin{gathered}
\left(\mathcal{A}_{\mu}(\tau)\right)^{-1}-\left(\mathcal{A}_{\mu}^{0}(\tau)\right)^{-1}-\left(\mathcal{K}_{\mu}(\tau)-\mathcal{L}_{\mu}(\tau)\right) \mathcal{P}_{1}-\mathcal{P}_{1}\left(\mathcal{K}_{\mu}(\tau)^{+}-\mathcal{L}_{\mu}(\tau)^{+}\right)^{*} \\
=\mathcal{V}_{\mu}(\tau)+\left(\mathcal{P}_{1}^{\perp}\left(\mathcal{A}_{\mu}(\tau)^{+}\right)^{-1}-\mathcal{P}_{1}^{\perp}\left(\mathcal{A}_{\mu}^{0}(\tau)^{+}\right)^{-1}-\mathcal{K}_{\mu}(\tau)^{+}\right)^{*}
\end{gathered}
$$


(recall that $\mathcal{V}_{\mu}(\tau)$ is the operator on the left side of (67)). Since Theorem 5 holds true for $\mathcal{A}_{\mu}(\tau)^{+}$, it follows that

$$
\left\|\mathcal{P}_{1}^{\perp}\left(\mathcal{A}_{\mu}(\tau)^{+}\right)^{-1}-\mathcal{P}_{1}^{\perp}\left(\mathcal{A}_{\mu}^{0}(\tau)^{+}\right)^{-1}-\mathcal{K}_{\mu}(\tau)^{+}\right\|_{\mathbf{B}\left(L_{2}(\Omega)\right)} \lesssim 1
$$

where we have used Poincaré's inequality and the fact that $\mathcal{P}_{1} \mathcal{K}_{\mu}(\tau)^{+}=0$. Thus, we are left with estimating the operator $\mathcal{V}_{\mu}(\tau)$.

Fix $f, g \in L_{2}(\Omega)$. Let $u, U$ and $v^{+}$be as above, and let $u^{+}=\left(\mathcal{A}_{\mu}^{0}(\tau)^{+}\right)^{-1} g$ and $U^{+}=\mathcal{K}_{\mu}(\tau)^{+} g$. Then, by (67),

$$
\begin{aligned}
\left(\mathcal{V}_{\mu}(\tau) f, g\right)_{2, \Omega}= & -\left(\mathcal{S}(\tau) \mathcal{P}_{1} u+\mathcal{T}(\tau) U, \mathcal{P}_{1}^{\perp}\left(v^{+}-U^{+}\right)\right)_{2, \Omega} \\
& -\left(U,\left(\mathcal{S}(\tau)^{+}+\mathcal{T}(\tau)^{+}\right)\left(v^{+}-\mathcal{P}_{1} u^{+}-U^{+}\right)\right)_{2, \Omega} \\
& -\left(U, \mathcal{S}(\tau)^{+} U^{+}-\varepsilon^{2} \bar{\mu} v^{+}\right)_{2, \Omega} .
\end{aligned}
$$

We use 73 , with $v^{+}-U^{+}$in place of $w^{+}$, to estimate, dropping the constants, the first expression on the right-hand side by

(75) $\left(\left\|\mathcal{D}_{2}(\tau) u\right\|_{1_{2}, 2, \Omega ; \tau}+|\tau|\|u\|_{1_{2}, 2, \Omega ; \tau}+\left\|\mathcal{D}_{1} U\right\|_{1_{2}, 2, \Omega ; \tau}\right)\left\|\mathcal{D}_{1}(\tau) \mathcal{P}_{1}^{\perp}\left(v^{+}-U^{+}\right)\right\|_{2, \Omega}$.

The remaining terms, according to estimates similar to $(58)$ and $(59)$ as well as Poincaré's inequality, do not exceed

(76) $\left\|\mathcal{D}_{1} U\right\|_{1_{2}, 2, \Omega ; \tau}\left(\left\|v^{+}-\mathcal{P}_{1} u^{+}-U^{+}\right\|_{1,2, \Omega ; \tau}+\left\|\mathcal{D}_{1} U^{+}\right\|_{1_{2}, 2, \Omega ; \tau}+|\tau|\left\|\mathcal{D}_{1}(\tau) v^{+}\right\|_{2, \Omega}\right)$

(notice here that $\mathcal{P}_{1} \mathcal{K}_{\mu}(\tau)=0$ ). Further, by Poincaré's inequality,

$$
\left\|\mathcal{D}_{1}(\tau) \mathcal{P}_{1}^{\perp}\left(v^{+}-U^{+}\right)\right\|_{2, \Omega} \lesssim\left\|\mathcal{D}_{1}(\tau)\left(v^{+}-u^{+}-U^{+}\right)\right\|_{2, \Omega}+\left\|\mathcal{D}_{1}(\tau) \mathcal{D}_{1}(\tau) u^{+}\right\|_{2, \Omega}
$$

and

$$
\begin{aligned}
\left\|v^{+}-\mathcal{P}_{1} u^{+}-U^{+}\right\|_{1,2, \Omega ; \tau} \lesssim & \left\|\mathcal{D}_{1}(\tau)\left(v^{+}-u^{+}-U^{+}\right)\right\|_{2, \Omega}+\left\|v^{+}-u^{+}\right\|_{1_{2}, 2, \Omega ; \tau} \\
& +\left\|\mathcal{D}_{1}(\tau) u^{+}\right\|_{1,2, \Omega ; \tau}+\left\|\mathcal{D}_{1} U^{+}\right\|_{1_{2}, 2, \Omega ; \tau}
\end{aligned}
$$

If we combine (75) with (77) and (76) with 78 and apply Lemmas 4 and 5 (for $\mathcal{K}_{\mu}(\tau)$ and $\mathcal{K}_{\mu}(\tau)^{+}$) and Theorems 4 and 5 (for $\mathcal{A}_{\mu}(\tau)^{+}$), then we obtain

$$
\left|\left(\mathcal{V}_{\mu}(\tau) f, g\right)_{2, \Omega}\right| \lesssim\|f\|_{2, \Omega}\|g\|_{2, \Omega} .
$$

This proves the theorem.

Acknowledgment. The author is grateful to T. A. SusLina for useful conversations about the problem. He also would like to thank V. P. SMYshLYAev for his interest in this work.

\section{REFERENCES}

[Al] G. Allaire, Homogenization and two-scale convergence, SIAM J. Math. Anal., 23 (1992), pp. 1482-1518.

[BP] N. Bakhvalov and G. Panasenko, Homogenisation: Averaging Processes in Periodic Media: Mathematical Problems in the Mechanics of Composite Materials, Nauka, Moscow, 1984 (in Russian); Kluwer Academic, Dordrecht, 1989 (in English).

[BlP] A. Bensoussan, J.-L. Lions and G. Papanicolaou, Asymptotic Analysis for Periodic Structures, North-Holland, Amsterdam, 1978.

[BSu1] M. Birman And T. Suslina, Threshold effects near the lower edge of the spectrum for periodic differential operators of mathematical physics, in Systems, Approximation, Singular Integral Operators, and Related Topics, Alexander A. Borichev and Nikolai K. Nikolski, eds., Birkhäuser, Basel, 2001, pp. 71-107. 
[BSu2] M. Sh. Birman and T. A. Suslina, Second order periodic differential operators. Threshold properties and homogenization, Algebra i Analiz, 15 (2003), no. 5, pp. 1-108 (in Russian); St. Petersburg Math. J., 15 (2004), pp. 639-714 (in English).

[BSu3] M. Sh. Birman and T. A. Suslina, Homogenization with corrector term for periodic elliptic differential operators, Algebra i Analiz, 17 (2005), no. 6, pp. 1-104 (in Russian); St. Petersburg Math. J., 17 (2006), pp. 897-973 (in English).

[BSu4] M. Sh. Birman and T. A. Suslina, Homogenization with corrector for periodic differential operators. Approximation of solutions in the Sobolev class $H^{1}\left(\mathbb{R}^{d}\right)$, Algebra i Analiz, 18 (2006), no. 6, pp. 1-130 (in Russian); St. Petersburg Math. J., 18 (2007), pp. 857-955 (in English).

[Bo] D. I. Borisov, Asymptotics for the solutions of elliptic systems with rapidly oscillating coefficients, Algebra i Analiz, 20 (2008), no. 2, pp. 19-42 (in Russian); St. Petersburg Math. J., 20 (2009), pp. 175-191 (in English).

[BCSu] R. Bunoiu, G. Cardone and T. Suslina, Spectral approach to homogenization of an elliptic operator periodic in some directions, Math. Meth. Appl. Sci., 34 (2011), pp. 10751096.

[ChC] K. D. Cherednichenko and S. Cooper, Resolvent estimates for high-contrast elliptic problems with periodic coefficients, Arch. Ration. Mech. Anal., to appear.

[GR1] G. GRISO, Error estimate and unfolding for periodic homogenization, Asymptot. Anal., 40 (2004), pp. 269-286.

[GR2] G. Griso, Interior error estimate for periodic homogenization, Anal. Appl., 4 (2006), pp. 61-79.

[KLS] C. E. Kenig, F. Lin and Z. Shen, Convergence Rates in $L_{2}$ for elliptic homogenization problems, Arch. Ration. Mech. Anal., 203 (2012), pp. 1009-1036.

[LnW] D. Lukkassen, G. Nguetseng and P. Wall, Two-scale convergence, Int. J. Pure Appl. Math., 2 (2002), pp. 35-86.

[MSh] V. G. Maz'ya and T. O. Shaposhnikova, Theory of Sobolev Multipliers: With Applications to Differential and Integral Operators, Springer, Berlin, 2009.

[OShY] O. A. Oleinik, A. S. Shamaev and G. A. Yosifian, Mathematical Problems in Elasticity and Homogenization, North-Holland, Amsterdam, 1992.

[PSu] M. A. Pakhnin And T. A. Suslina, Operator error estimates for homogenization of the elliptic Dirichlet problem in a bounded domain, Algebra i Analiz, 24 (2012), no. 6, pp. 139-177 (in Russian); St. Petersburg Math. J., 24 (2013), pp. 949-976 (in English).

$[\mathrm{P}] \quad$ S. E. PAstuknova, Approximations of the resolvent for a non-self-adjoint diffusion operator with rapidly oscillating coefficients, Mat. Zametki, 94 (2013), pp. 130-150 (in Russian); Math. Notes, 94 (2013), pp. 127-145 (in English).

[PT] S. E. Pastukhova And R. N. Tiknomirov, Operator estimates in reiterated and locally periodic homogenization, Dokl. Acad. Nauk, 415 (2007), pp. 304-309 (in Russian); Dokl. Math., 76 (2007), pp. 548-553 (in English).

[SE1] N. N. SENIK, Homogenization for a periodic elliptic operator in a strip with various boundary conditions, Algebra i Analiz, 25 (2013), no. 4, pp. 182-259 (in Russian); St. Petersburg Math. J., 25 (2014), pp. 647-697 (in English).

[SE2] N. N. SENIK, On homogenization for non-self-adjoint periodic elliptic operators on an infinite cylinder, Funktsional. Anal. i Prilozhen., 50 (2016), no. 1, to appear (in Russian).

[Su1] T. A. Suslina, On homogenization for a periodic elliptic operator in a strip, Algebra i Analiz, 16 (2004), no. 1, pp. 269-292 (in Russian); St. Petersburg Math. J., 16 (2005), pp. 237-257 (in English).

[Su2] T. A. Suslina, Homogenization of a stationary periodic Maxwell system, Algebra i Analiz, 16 (2004), no. 5, pp. 162-244 (in Russian); St. Petersburg Math. J., 16 (2005), pp. 863-922 (in English).

[Su3] T. A. Suslina, Homogenization in the Sobolev class $H^{1}\left(\mathbb{R}^{d}\right)$ for second order periodic elliptic operators with the inclusion of first order terms, Algebra i Analiz, 22 (2010), no. 1, pp. 108-222 (in Russian); St. Petersburg Math. J., 22 (2011), pp. 81-162 (in English).

[Su4] T. A. Suslina, Homogenization of elliptic systems with periodic coefficients: operator error estimates in $L_{2}\left(\mathbb{R}^{d}\right)$ with corrector taken into account, Algebra i Analiz, 26 (2014), no. 4, pp. 195-263 (in Russian); St. Petersburg Math. J., 26 (2015), pp. 643-693 (in English). 
[S-HT] J. Sanchez-Hubert and N. Turbe, Ondes élastiques dans une bande périodique, Modél. Math. Anal. Numér., 20 (1986), pp. 539-561.

[ZhKO] V. V. Zhikov, S. M. Kozlov and O. A. Oleinik, Homogenization of differential operators and integral functionals, Nauka, Moscow, 1993 (in Russian); Springer, Berlin, 1994 (in English).

[ZH] V. V. Zhikov, On operator estimates in homogenization theory, Dokl. Acad. Nauk, 403 (2005), pp. 305-308 (in Russian); Dokl. Math., 72 (2005), pp. 535-538 (in English).

[ZhP] V. V. Zhikov and S. E. Pastukhova, On operator estimates for some problems in homogenization theory, Russ. J. Math. Phys., 12 (2005), pp. 515-524. 